\title{
Self-Identifying Data for Fair Use
}

\section{Citation}

Chong, Stephen, Christian Skalka, and Jeffrey A. Vaughan. 2015. “Self-Identifying Data for Fair Use." J. Data and Information Quality 5 (3) (March 2): 1-30. doi:10.1145/2687422.

\section{Published Version}

doi:10.1145/2687422

\section{Permanent link}

http://nrs.harvard.edu/urn-3:HUL.InstRepos:22043260

\section{Terms of Use}

This article was downloaded from Harvard University's DASH repository, and is made available under the terms and conditions applicable to Open Access Policy Articles, as set forth at http:// nrs.harvard.edu/urn-3:HUL.InstRepos:dash.current.terms-of-use\#OAP

\section{Share Your Story}

The Harvard community has made this article openly available.

Please share how this access benefits you. Submit a story.

Accessibility 


\section{Self-Identifying Data for Fair Use}

STEPHEN CHONG, Harvard University

CHRISTIAN SKALKA, The University of Vermont

JEFFREY A. VAUGHAN, Google

Public-use earth science datasets are a useful resource with the unfortunate feature that their provenance is easily disconnected from their content. "Fair-use policies" typically associated with these datasets require appropriate attribution of providers by users, but sound and complete attribution is difficult if provenance information is lost. To address this we introduce a technique to directly associate provenance information with sensor datasets. Our technique is similar to traditional watermarking but is intended for application to unstructured time-series datasets. Our approach is potentially imperceptible given sufficient margins of error in datasets, and is robust to a number of benign but likely transformations including truncation, rounding, bit-flipping, sampling, and reordering. We provide algorithms for both one-bit and blind mark checking, and show how our system can be adapted to various data representation types. Our algorithms are probabilistic in nature and are characterized by both combinatorial and empirical analyses. Mark embedding can be applied at any point in the data lifecycle, allowing adaptation of our scheme to social or scientific concerns.

Categories and Subject Descriptors: E.m [Data]: Miscellaneous; H.3.m [Information Systems]: Information Storage and Retrieval-Miscellaneous

General Terms: Design, Documentation, Reliability.

Additional Key Words and Phrases: Provenance, Self-identifying data

ACM Reference Format:

ACM J. Data Inform. Quality V, N, Article A (January YYYY), 30 pages.

DOI : http://dx.doi.org/10.1145/0000000.0000000

\section{INTRODUCTION}

Open sharing of datasets is beneficial for scientific research, but is often complicated by issues related to attribution, i.e. who should be acknowledged, and how, if a particular dataset is used? Two major problems present themselves: how to associate provenance metadata with datasets, and what is the nature of metadata. In environmental sciences datasets in particular, dataset usage often brings with it an informal obligation to attribute organizations that manage areas from which data is collected, e.g. field stations, as well as collectors of data, e.g. University research groups. These are typically called "fair use policies", and different datasets are subject to different, possibly complex policies. And although standards such as eml [Arndt et al. 2000] have been proposed, different repositories use different techniques to associate data and metadata such as provenance information. Even if researchers are well-intentioned, metadata and datasets can become disassociated during usage, and provenance information lost, leading to incomplete or inaccurate attributions in publications and other research products.

Permission to make digital or hard copies of part or all of this work for personal or classroom use is granted without fee provided that copies are not made or distributed for profit or commercial advantage and that copies show this notice on the first page or initial screen of a display along with the full citation. Copyrights for components of this work owned by others than ACM must be honored. Abstracting with credit is permitted. To copy otherwise, to republish, to post on servers, to redistribute to lists, or to use any component of this work in other works requires prior specific permission and/or a fee. Permissions may be requested from Publications Dept., ACM, Inc., 2 Penn Plaza, Suite 701, New York, NY 10121-0701 USA, fax +1 (212) 869-0481, or permissions@acm.org.

(c) YYYY ACM 1936-1955/YYYY/01-ARTA $\$ 15.00$

DOI : http://dx.doi.org/10.1145/0000000.0000000 
In this paper, we explore a scheme for embedding a provenance identifier in environmental datasets, that associates metadata with datasets in a tightly coupled manner that does not rely on external structure such as XML formats or database schema. We say that such datasets are "self identifying". Further, this identifier provides a level of indirection allowing referenced metadata to be structured and organized in an arbitrary manner. For example, the identifier could be a URL link to a data object with extensive provenance information. Our technique is suited to environmental datasets because it exploits noise common to instruments used in environmental sciences data gathering, in particular, margin of error in instrumentation. It can be viewed as a type of watermarking scheme, where the watermark does not alter data beyond the bounds of its error bars, and is thus "imperceptible" by data end-users, but is nevertheless retrievable by algorithmic means. And while these provenance identifiers are not impervious to attack as would be a security mechanism, they are robust to corruptions-including reordering, sampling, truncation, and rounding - that can be expected during the course of normal dataset analysis by end users. Thus, our approach is well-suited to supporting attribution and fair use policies in environmental datasets.

\subsection{Provenance Connections}

There is a growing body of literature on provenance issues in informatics, and the Open Provenance Model [Moreau et al. 2011] even provides a structured format and ontology for provenance information in data systems. Our provenance identifiers are intended to refer to provenance information, and in this sense the relation of our work to provenance is indirect. However, we aim to address a significant issue with respect to provenance in practice, which is adaptation and use of provenance schemes. Rather than associating provenance metadata with datasets via e.g. XML data structure that may be discarded and lost by data users, provenance identifiers are "baked in" to the data itself, and are robust to certain expected transformations. At the same time, references to provenance information can be used to easily retrieve the latter when needed e.g. via web tools, as we discuss in Section 8. By alleviating end-users from the burden of maintaining metadata structure during use, we argue that our scheme will promote more reliable use of provenance information.

\subsection{A Proposed Application Setting}

The Sagehen Creek Field Station (CA) provides both a setting for environmental field research and data gathering, and an online data repository for associated datasets. On its data repository web portal [Sagehen Creek Field Station Data Repository ], a blanket fair use policy is stated requiring acknowledgment of the field station and data owners, and a request to contact data owners to discuss attribution details. Similarly, the Hubbard Brook Experimental Forest $(\mathrm{NH})$ also provides both a research setting and online repository [Hubbard Brook Ecosystem Study ]. In this case, specific fair use policies are associated with individual datasets (although a general default policy appears to cover most), and users must electronically sign a fair use agreement prior to downloading data. In any case it is clear that use policies are sufficiently complex that repositories must clearly enunciate them, and encourage compliance with them. And in fact, although research scientists are typically well-intentioned, anecdotal evidence from station managers suggests that fair use policies are often violated.

We argue that these violations are often caused by the decoupling of datasets and their provenance information during normal scientific usage. Once datasets are downloaded, they are often reformatted, entered into local databases with arbitrary schema, and passed around between users. Policies that were reviewed when data was downloaded may be forgotten or not communicated between users, and data structure im- 
posed in XML or similar forms may be discarded once datasets are extracted from them, and metadata lost. We envision that our scheme can be used to mark datasets intended for public use, for example by data repository managers. This mark, when recovered from datasets, can be interpreted as a pointer to online provenance information and fair use policies. Thus, any end-user, or reader of their work, could copy-paste a subset of their dataset into a recovery tool and be immediately directed to relevant provenance information for the dataset. The scheme therefore provides much greater ease of access to provenance information, and user accountability for proper attribution.

It is important to note that the addition of a provenance mark does alter datasets, however imperceptibly. But an appealing feature of our scheme is that a provenance mark can be applied at any point in the data lifecycle. Thus, marking strategies can be flexible, and unmarked, raw datasets can be retained by data producers, or made available on repositories along with marked datasets. This will allow data providers to negotiate marking strategies with repository managers, and determine the best tradeoff between ease of attribution and fidelity to original data. In most cases, we expect that marks will be applied post-processing, e.g. after raw sensor voltage readings are converted to physical units and smoothed. In any case, in Section 7 we provide an analysis that establishes quantitative guidelines for use of the system, e.g. expected dataset entropy.

To provide a user experience that actively illustrates these ideas, we have developed a prototype application, described in Section 8 and available online. It shows how data providers can embed provenance marks in datasets without significant alterations to existing web interfaces or data distribution schemes. It also illustrates how users of datasets can easily retrieve associated metadata from a sampling of datapoints. Furthermore, the application shows how data can be marked post-processing, and that marking does not preclude access to unmarked datasets.

\subsection{Technical Background and Contributions}

Watermarking refers to any number of techniques for marking a real or virtual item with some sort of provenance identifier, in a manner that does not interfere with normal use of the item and is "indelible" by some measure. An analog example is the dollar bill, which contains a watermark that is visible when held against a bright light, using a technique developed in the 13th century to protect against counterfeiting. In the digital realm, it has been adapted to audio and video data, wherein signals are altered to carry the mark in subtle ways that are undetectable by human senses. In contrast to analog watermarking, digital watermarking usually does not prevent copying of data, but ensures that copying retains the watermark and thus encoded provenance information.

Watermarking of digital media is typically treated as a signal processing issue [Cox and Miller 2002]. Watermarking techniques have also been studied in application to relational databases [Agrawal and Kiernan 2002] and streaming data [Sion et al. 2006]. Usually these approaches leverage the database schema, or the sequential structure of a data stream, in order to embed watermarks in the data itself.

Our main contribution is a new watermarking technique for environmental sciences data. Similar to database watermarking it is combinatorial in nature, and allows typical datasets to be marked in a manner that does not interfere with normal scientific use. But unlike database watermarking, the technique makes minimal assumptions about the structure of datasets. While it is not a security mechanism per se, it is robust to corruptions including reordering, sampling, truncation, and rounding, which we argue is sufficient to support fair use policies in the scientific user community. 
We have evaluated our system from both empirical and analytic perspectives. Using a prototype implementation of our system and a simulation of watermarked dataset corruptions, we have characterized the robustness of our approach in practice. And, a combinatorial analysis yields an upper bound probability for recovering a watermark from a dataset with our technique. Results obtained from these exercises demonstrate its flexibility and resilience. Further underscoring the practical applicability of our approach, we formulate a general method for adapting it to a variety of data representation types, e.g. integer values, floating point values, and geographic datapoints.

To illustrate how our system can be used in practice to support fair use policies, we have applied our method to encode a 32-bit provenance mark in an existing public use dataset, available online. We have also made available online an interface for extracting 32-bit provenance marks and reporting provenance information associated with marked datasets. URLs for these tools, and further discussion of them, are provided in Section 8.

\subsection{Characterization of the Technique}

As described above, our goal is to design a system that can embed provenance information into, and retrieve such information from, scientific datasets. Furthermore our techniques are intended to be robust against certain natural, non-adversarial dataset corruptions. The remainder of the paper comprises a detailed description and analysis of our embedding and retrieval algorithms; now we provide a high-level characterization of our technique, using common parlance of the watermarking literature for clarity. In particular, we classify our system under the standard categories of perceptibility, robustness, and capacity [Fridrich and Goljan 1999].

Perceptibility. This classification refers to whether a watermark can be "perceived" in the marked object; its meaning is media-dependent and defined with respect to human perception. Since watermarking has not been previously studied in the context of environmental datasets, we propose a definition of perceptibility in this context, under the assumption that any dataset is a collection of numeric values, with possibly significant ordering. To guide intuition, we (informally) assume that individual data points have a precision that is coarser than the precision of the datatypes used to represent the numeric values. We say that a watermark is imperceptible in a dataset if the watermark does not inhibit standard uses of the dataset. Specifically, watermarking should not significantly affect the scientific use of the dataset. Consider, as an example, the Sensirion SHT11 temperature sensor, which is accurate to at best $\pm 0.5^{\circ} \mathrm{C}$. Altering data produced by a Sensirion SHT11 by amounts orders of magnitude smaller than $0.5^{\circ} \mathrm{C}$ does not affect use of that data, since such alterations are well within the sensor data error.

Robustness. Robustness refers to how well a watermark stands up to transformations of data, benign or malicious. Our scheme is semi-fragile, in the sense that it is capable of withstanding many, but not all, transformations. It is not intended as a security mechanism per se; rather our goal is to withstand benign transformations that scientists might impose in the natural course of dataset usage. These transformations include truncation and quantization (rounding) of digits, as well as random bit flips. Our scheme is also robust to sampling of datasets, i.e. where a new dataset is generated by a selection of datapoints from the original dataset; this is also called a subset attack in the database watermarking literature [Agrawal and Kiernan 2002]. Reordering of data within the dataset also has no effect on the reliability of our mechanisms.

Capacity. This category refers to whether a mechanism allows to check if an object is marked with a given watermark (so-called one-bit watermarking), or whether it allows a watermark to be extracted from an object with no prior knowledge of the embedded watermark (so-called blind watermarking). Since we expect both capacities 
to support the user community, we define techniques for both one-bit and blind marking of datasets. Indeed, our algorithm for the latter relies on the former for increased probability of correct mark extraction.

\subsection{Outline of the Paper}

The remainder of the paper is structured as follows. In Section 2 we provide a technical problem statement, define our corruption model, and summarize our embedding and retrieval technique at a high level. In Section 3 we describe our embedding algorithm in detail. In Section 4 we define our one-bit checking technique, and in Section 5 we define our blind retrieval algorithm. In both of these Sections we discuss how our approach is robust to various corruptions. Our basic theory treats datasets as sequences of bit vectors representing natural numbers; in Section 6 we formulate a methodology for extending our approach to various datatypes such as integers, doubles, and GPS coordinates. In Section 7 we report results of combinatorial analysis and empirical tests that illustrate robustness of our technique. We have developed a prototype implementation of our technique, which is deployed as a web application, allowing consumers of self-identifying datasets to easily check or discover provenance information. We describe the implementation in Section 8. In Section 9 we summarize deployment issues and how we might address them in future work. We discuss related work in Section 10, and conclude in Section 11.

This paper substantially revises earlier work by same authors [Chong et al. 2010]. The present paper contains an greatly expanded analysis of non-integral data including floating point and GPS values, describes the implementation of a new prototype web application that decodes concise provenance identifiers from datasets and associates those identifiers with descriptive provenance text, discusses the application of this tool to an environmental dataset, and reports on additional experimental results with both real GPS data and synthetic data.

\section{PROBLEM DESCRIPTION AND TECHNICAL SUMMARY}

In this section we provide description of the problem of interest, and also an informal description of how we address it. For simplicity we will consider bit vector representations of data, where bitvec $_{n}$ denotes the set of bit vectors of length $n$ and bitvec represents the set of all finite length bit vectors.

\subsection{Problem Statement}

A provenance mark (or simply, mark) is a bit vector of fixed length $L_{m}$. We use syntax mark $\equiv \operatorname{bitvec}_{L_{m}}$ for the set of all marks. Intuitively a mark is an identifier that may refer to more elaborate metadata via some standard such as DOI. In this paper, we are not concerned with the allocation of provenance marks, or how a provenance mark is linked to more detailed metadata. We assume that the length of provenance marks $L_{m}$ is universally agreed upon and known.

A datapoint is a bit vector of possibly variable length, and the set of datapoints is written datapoint $\equiv$ bitvec. Datasets, lists of datapoints, may be subject to various forms of corruption, for example rounding or dropping datapoints (see the following section). We model dataset corruption with functions of form list(datapoint) $\rightarrow$ list(datapoint).

Letting $\times$ denote the cartesian product, our goal is to implement the functions

$$
\begin{aligned}
& E \text { : } \operatorname{list}(\text { datapoint }) \times \text { mark } \rightarrow \operatorname{list}(\text { datapoint }) \\
& R: \text { list(datapoint) } \rightarrow \text { mark } \\
& C: \text { list (datapoint) } \times \text { mark } \rightarrow\{\text { True, False }\}
\end{aligned}
$$


that respectively encode a provenance mark into data set, retrieve an encoded provenance mark from a dataset, and check that mark is present in a dataset. $\mathrm{s}$

It is normal that sometimes mark retrieval or checking will fail. This may occur when a dataset is too small or corruption to too severe. The following equations characterize successful instances of retrieval and checking:

$$
\begin{aligned}
R(f(E(\mathcal{D S}, m))) & =m \\
C\left(f(E(\mathcal{D S}, m)), m^{\prime}\right) & = \begin{cases}\text { True } & \text { when } m=m^{\prime} \\
\text { False } & \text { when } m \neq m^{\prime}\end{cases}
\end{aligned}
$$

where $\mathcal{D S}: \operatorname{list}\left(\right.$ datapoint) and $m, m^{\prime}:$ mark and $f: \operatorname{list}$ (datapoint) $\rightarrow \operatorname{list}($ datapoint) models dataset corruption.

Notation. For bit vector $v \in$ bitvec, we write $|v|$ for the length (size) of $v$, and write $\mathrm{v}_{i}$ to refer to the $i$ th bit of $\mathrm{v}$, where $i=0$ is the most significant (left-most) bit and $i=|\mathrm{v}|-1$ is the least significant (right-most) bit. We write $\mathrm{v} @ \mathrm{v}^{\prime}$ for the concatenation of bit vectors $v$ and $v^{\prime}$. Finally, we write $|L|$ for the length of list $L$.

\subsection{Corruption Model}

In our corruption model we allow the following transformations:

- Rounding. Datapoints are rounded to the nearest multiple of some integer $n$.

- Truncation. Some number of least-significant bits may be removed from any datapoint in the given dataset. ${ }^{1}$

- Deletion / Sampling. Datapoints may be removed from the given dataset.

- Reordering. Datapoints in a dataset may be permuted.

- Bit flip. One or more bits of any datapoint in the given dataset may be changed.

We assume that truncation, rounding, sampling, and reordering are more likely to occur than bit flips. For example, copying data (for example, from one spreadsheet to another) is more likely to truncate or round data values than it is to randomly flip bits. Note that rounding and truncation tend to corrupt or remove the less significant bits from datapoints. That is, more significant bits in datapoints are more likely to be unaffected by truncation and rounding. Our embedding and retrieval processes are designed to take advantage of this behavior.

There are of course limits on the robustness of any pair of encoding and retrieval functions within this corruption model. For example removing all datapoints from a dataset will disallow retrieval. Similarly, replacing the entire dataset with random data through bit flips will also prevent retrieval.

\subsection{Informal Summary of the Technique}

Our technique embeds a provenance mark in the lower-order bits of datapoints in a dataset. The embedding is designed to be robust to corruption in the lower-order bits of datapoints. That is, our technique assumes that lower-order bits are more likely to be corrupted than higher-order bits.

To support one-bit checking (wherein given dataset $\mathcal{D S}$ and provenance mark $\mathrm{m}$, we want to answer the question "was m embedded in $\mathcal{D S}$ ?") we use two of the lowerorder bits of each datapoint in the dataset as check bits. One of the check bits is used to help the retrieval process determine the parameters used for the embedding; the other check bit is a hash of the encoded provenance mark concatenated with the more significant bits of the datapoint. Given sufficiently many datapoints whose check bits

\footnotetext{
${ }^{1}$ We do not regard truncation as a special case of rounding. Truncation of the least-significant $k$ bits will affect only $k$ bits; rounding to the nearest $n=2^{k}$ may affect an arbitrary number of bits. For example, $10111(=23)$ rounded to the nearest 4 is $11000(=24)$, with 5 bits affected.
} 
(and more significant bits) are uncorrupted, the encoding parameters can be retrieved, and one-bit checking supported.

To support blind checking (wherein given dataset $\mathcal{D S}$, we want to answer the question "which provenance mark was embedded in $\mathcal{D S}$ ?"), our encoding technique breaks a provenance mark into a number of smaller pieces, and replaces the least significant bits of each datapoint with one of these provenance pieces. We leverage redundancy, allowing a bit of the provenance mark to appear in more than one provenance piece, which provides greater robustness to truncation and rounding. The significant bits of a datapoint are used to determine which provenance piece replaces the least significant bits. During retrieval, the least significant bits of the datapoints are examined to retrieve a "best guess" at the provenance mark, which can then be tested using one-bit checking. If the best guess is incorrect, the retrieval process permits limited search of possible provenance marks, where more likely marks are considered first.

\section{EMBEDDING A PROVENANCE MARK IN A DATASET}

In this section we describe how we embed a provenance mark m of length $L_{m}$ into a dataset. We assume that each datapoint in the dataset contains insignificant bits: a non-zero number of least significant bits that can be safely manipulated within the error bounds of the device that generated the data. We embed a provenance mark into a dataset by encoding metadata into the insignificant bits of each datapoint. We further assume that each datapoint in a dataset is the same length, contains the same number of insignificant bits, and represents non-negative integers. If datapoints are of different length, we can, without loss of generality, pad datapoints with leading zeros until they are the same length. The number of insignificant bits is inherent in the physical characteristics of the sensor generating the dataset, and so all datapoints in the dataset should have an identical number of insignificant bits. In Section 6 we describe how our technique can be generalized to floating point and negative values.

Let $L_{m d}$ denote the number of insignificant bits in a datapoint, and $L_{s i g}$ denote the number of significant bits; thus the length of any datapoint is $L_{m d}+L_{s i g}$. Clearly the values of $L_{m d}$ and $L_{s i g}$ are dependent on the given dataset, since the number of significant bits is a property of the sensors used to collect the data.

We refer to a datapoint in which the insignificant bits have been replaced with metadata as an annotated datapoint. The process of embedding a provenance mark in a dataset produces an uncorrupted annotated dataset containing uncorrupted annotated datapoints.

Figure 1 shows the structure of an annotated datapoint. The $L_{m d}$ insignificant bits have been replaced with a parameter check bit, a mark check bit, and a provenance piece. We thus require that each datapoint contains at least 3 insignificant bits (i.e., $L_{m d} \geq 3$ ). The provenance piece is a subset of bits of the provenance mark; Section 3.1 describes how provenance pieces are derived from a provenance mark. The parameter check bit and mark check bit are derived from the datapoint's significant bits and the parameters for the embedding, described in Section 3.2. The check bits enable the retrieval process to verify, with high probability, which provenance mark was embedded in the dataset.

\subsection{Provenance Pieces}

Each annotated datapoint contains $L_{m d}-2$ bits of the provenance mark, referred to as a provenance piece. If the length of the provenance mark $L_{m}$ is greater than $L_{m d}-2$, then a single datapoint cannot contain all bits of the provenance mark. The embedding process chooses provenance pieces to ensure that an annotated dataset contains all bits of the provenance mark with high probability. 


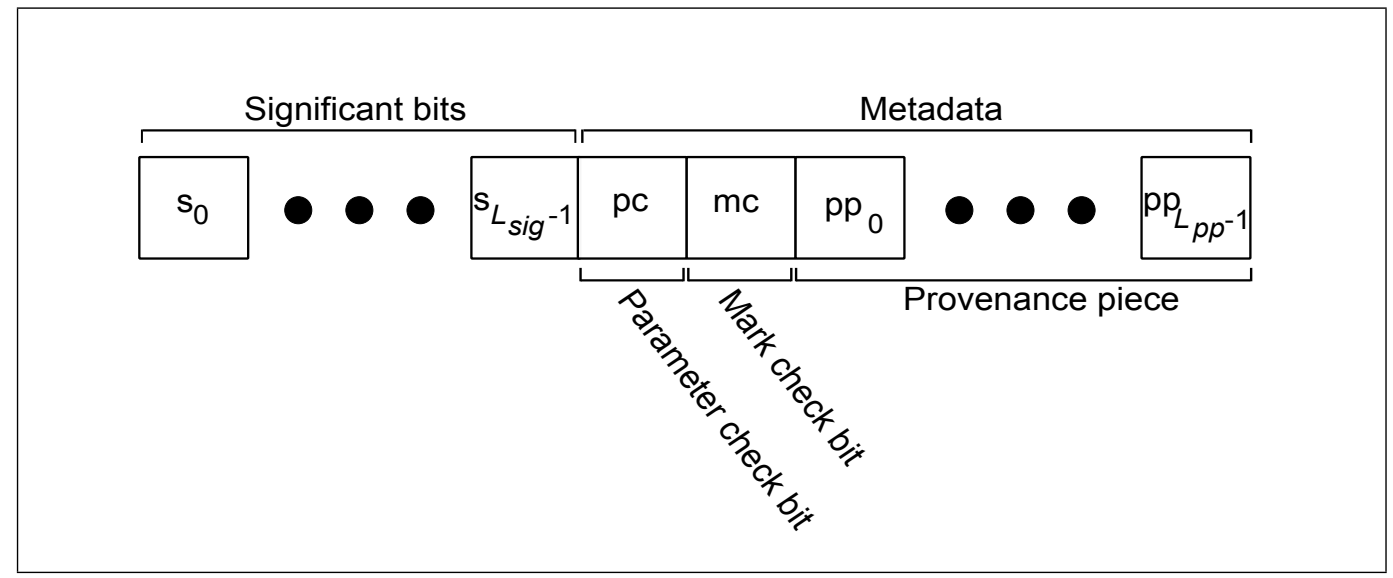

Fig. 1. Anatomy of an Annotated Datapoint

The embedding process takes as a parameter $N_{p p}$, the number of distinct provenance pieces. For each datapoint one of the $N_{p p}$ provenance pieces is chosen to be embedded into it. Specifically, given a datapoint with significant bits $\mathrm{s}$, we choose the $k$ th provenance piece, where $k=\operatorname{hash}\left(N_{p p}, \mathrm{~s}\right)$ and $\operatorname{hash}(n, \mathrm{v})$ is a cryptographic hash of bit vector $\checkmark$ that returns a value in $\mathbb{Z}_{n}$. Provided that a dataset has sufficient variation in the significant bits of its constituent datapoints, the $N_{p p}$ provenance pieces will be embedded uniformly at random throughout the dataset. Thus, any sufficiently large random subset of an annotated dataset is likely to include every distinct provenance piece. This supports robustness in the retrieval process. Section 6 discusses an extension to this technique that treats low entropy datasets.

Given provenance mark $\mathrm{m}$ of length $L_{m}$, and given the number of provenance pieces $N_{p p}$ and insignificant bits $L_{m d}$, then the $N_{p p}$ distinct provenance pieces are defined as follows. Let $L_{p p}=L_{m d}-2$ be the length of each provenance piece. For $k \in 0 . .\left(N_{p p}-1\right)$ and $i \in 0 . .\left(L_{p p}-1\right)$, let the $i$ th bit of the $k$ th provenance piece, denoted $\mathrm{pp}_{i}^{k}$, be defined as

$$
\mathrm{pp}_{i}^{k}=\mathrm{m}_{j} \text { where } j=\left(k \frac{L_{m}}{N_{p p}}+i\right) \quad \bmod L_{m} .
$$

Note that a given bit of provenance mark $m$ may appear in more than one provenance piece. This redundancy means that not all distinct provenance pieces need to be available during the retrieval process. Furthermore, since the same mark bit may occur in a more significant position in one provenance piece than another, redundancy provides robustness to truncation and rounding.

Figure 2 gives an example of how a provenance mark of length 16 is split into four distinct provenance pieces of length 8 (i.e., $L_{m}=16, N_{p p}=4$, and $L_{p p}=8$ ). Note that each provenance piece contains 8 contiguous bits of the provenance mark. (The fourth provenance piece, $\mathrm{pp}^{3}$, contains the last four bits of $\mathrm{m}$ followed by the first 4 bits.) Note also that each bit of the provenance mark appears in exactly two provenance pieces. Thus, provenance mark $\mathrm{m}$ could be retrieved if the retrieval process has either provenance pieces $\mathrm{pp}^{0}$ and $\mathrm{pp}^{2}$, or provenance pieces $\mathrm{pp}^{1}$ and $\mathrm{pp}^{3}$. This redundancy means that a subset of the provenance pieces may suffice to retrieve the provenance mark. Furthermore, if a dataset was truncated by, say, a single bit, then the least significant bit of piece $\mathrm{pp}^{0}$ (corresponding to the 7 th bit of $\mathrm{m}$ ) would be lost from every instance, but the 7th bit of $\mathrm{m}$ would still be available in all instances of piece $\mathrm{pp}^{1}$. 


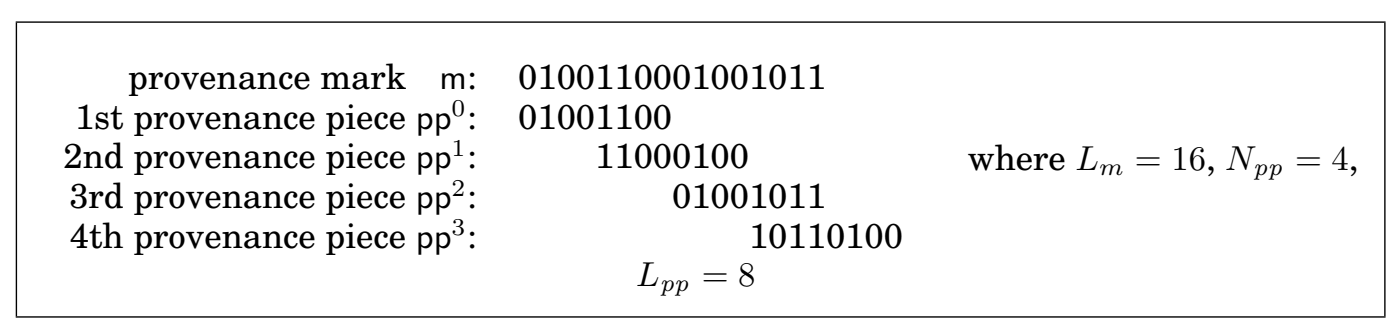

Fig. 2. Provenance pieces example.

To ensure that each bit of the provenance mark appears in the same number of distinct provenance pieces, we require that $N_{p p} \times L_{m}$ is divisible by $L_{p p}$. Moreover, since each provenance piece should contain distinct bits of the provenance mark, we require that $N_{p p} \leq L_{m}$.

\subsection{Parameter and Mark Check Bits}

The parameter check bit and mark check bit are derived from the encoding parameters, and are used in one-bit checking and during retrieval to determine with high probability if the correct provenance mark has been retrieved. The parameter check bit $p c$ for a datapoint with significant bits $s$ is computed by taking the hash of $s$ and the number of distinct provenance pieces, $N_{p p}$. The mark check bit mc is the hash of $\mathrm{s}$ and the provenance mark m. Formally, we have:

$$
\begin{aligned}
\mathrm{pc} & =\operatorname{hash}\left(2, \mathbf{s} @ N_{p p}\right) \\
\mathrm{mc} & =\operatorname{hash}(2, \mathbf{s} @ \mathrm{~m}) .
\end{aligned}
$$

Section 4 describes how the parameter and mark check bits are used by one-bit checking.

\section{CHECKING A PROVENANCE MARK}

The embedding process embeds a provenance mark $m$ into a dataset to produce an uncorrupted annotated dataset. As the dataset is disseminated and used, it may be corrupted, for example by datapoints being rounded or deleted as described in Section 2.2. However, we can use a corrupted annotated dataset for both one-bit watermarking and blind watermarking. In one-bit watermarking, we can determine with high probability whether a provenance mark $\mathrm{m}^{\prime}$ is the provenance mark $\mathrm{m}$ that was embedded into the dataset. Blind watermarking allows us to retrieve $m$ with high probability.

In this section, we describe how to perform one-bit watermarking using a corrupted annotated dataset. In Section 5 we describe how to perform blind watermarking, and our technique there relies on the one-bit checking described here to increase probabilities of correctness.

\subsection{Retrieving $L_{s i g}$ and $N_{p p}$}

The embedding process adds a parameter check bit to each datapoint to assist the retrieval of embedding parameters $L_{s i g}$ (the number of significant bits of a datapoint) and $N_{p p}$ (the number of distinct provenance pieces used in the embedding process). The retrieval process guesses values for $L_{s i g}$ and $N_{p p}$, and uses the parameter check bits to determine (with high probability) when it has guessed the values correctly.

A corrupted annotated datapoint $\mathrm{d}$ is defined to be pc-consistent with guesses $L_{\text {sig }}$ and $N_{p p}$ iff

$$
\operatorname{hash}\left(2, \mathrm{~d}_{0} \ldots \mathrm{d}_{L_{s i g}-1} @ N_{p p}\right)=\mathrm{d}_{L_{s i g}} .
$$


Note that if the guesses for $L_{s i g}$ and $N_{p p}$ are correct, and bits $\mathrm{d}_{0} \ldots \mathrm{d}_{L_{s i g}}$ have not been corrupted, then $\mathrm{d}_{L_{s i q}}$ is the parameter check bit as computed by Equation 1 . If the guesses for $L_{s i g}$ and $N_{p p}$ are incorrect, or one or more bits $\mathrm{d}_{0} \ldots \mathrm{d}_{L_{s i g}}$ have been corrupted, then the probability of the datapoint being pc-consistent is approximately $\frac{1}{2}$, due to the properties of the cryptographic hash function used to generate the parameter check bit.

We define the pc-consistency score of corrupted annotated dataset $\mathcal{D S}$ for guesses $L_{\text {sig }}$ and $N_{p p}$ to be the proportion of datapoints in $\mathcal{D S}$ that are pc-consistent for guesses $L_{\text {sig }}$ and $N_{p p}$. If the guesses are correct, and the first $L_{s i g}+1$ bits of each datapoint have not been corrupted, then the pc-consistency score will be 1 . If the guesses are incorrect, then (regardless of the corruption of the datapoints) the expected pc-consistency score is $\frac{1}{2}$. In general, the probability of an incorrect guess having pc-consistency score of 1 is $2^{-n}$, where $n=|\mathcal{D S}|$, which is vanishingly small for an annotated corrupted dataset $\mathcal{D S}$ of reasonable size.

Because guesses $L_{\text {sig }}$ and $N_{p p}$ are drawn from limited domains $(\{1 \ldots \max \{|\mathrm{d}| \mid$ $\mathrm{d} \in \mathcal{D S}\}\}$ and $\left\{1 \ldots L_{m}\right\}$ respectively) it is feasible to enumerate all possible guesses $\left(L_{s i g}, N_{p p}\right)$ and calculate their pc-consistency score. The parameters with the best pcconsistency score are used for the following step, checking a provenance mark. In Section 7 , we investigate the effects of corruption on the pc-consistency score.

\subsection{Checking a Provenance Mark}

Suppose we have a corrupted annotated dataset $\mathcal{D S}$ for which we know the embedding parameter $L_{s i g}$, and we have a guess at the provenance mark $\mathrm{m}$. (We describe in Section 5 how we retrieve one or more guesses for the provenance mark for blind checking.) The embedding process adds a mark check bit to each datapoint to determine with high probability when the correct provenance mark has been guessed.

Given a corrupted annotated datapoint $\mathrm{d} \in \mathcal{D S}$, we say that $\mathrm{d}$ is $m c$-consistent with $L_{s i g}$ and $\mathrm{m}$ iff

$$
\operatorname{hash}\left(2, \mathrm{~d}_{0} \ldots \mathrm{d}_{L_{s i g}-1} @ \mathrm{~m}\right)=\mathrm{d}_{L_{s i g}+1} .
$$

Note that if the guesses for $L_{\text {sig }}$ and $\mathrm{m}$ are correct, and the bits $\mathrm{d}_{0} \ldots \mathrm{d}_{L_{s i g}+1}$ have not been corrupted, then $\mathrm{d}_{L_{s i g}+1}$ is the mark check bit as computed by Equation 2. Otherwise, the probability of the datapoint being mc-consistent is approximately $\frac{1}{2}$.

The mc-consistency score of dataset $\mathcal{D S}$ for $L_{\text {sig }}$ and $\mathrm{m}$ is the proportion of datapoints in $\mathcal{D S}$ that are mc-consistent for $L_{\text {sig }}$ and $\mathrm{m}$. As with the pc-consistency score, if $L_{\text {sig }}$ and $\mathrm{m}$ are correct and the first $L_{\text {sig }}+2$ bits of each datapoint are uncorrupted, the mc-consistency score will be 1 , otherwise the expected mc-consistency score is $\frac{1}{2}$. In Section 7 , we investigate the effects of corruption on the mc-consistency score, and given an mc-consistency score, when that implies the provenance mark is correct.

\section{RETRIEVING THE PROVENANCE MARK}

In addition to the parameter check bit and mark check bit, the embedding process adds to each point in the dataset a provenance piece containing bits of the provenance mark $\mathrm{m}$. To retrieve a provenance mark from a corrupted annotated dataset (also known as blind watermarking), we extract provenance pieces from the dataset and combine them to construct a best guess at the provenance mark. Because the dataset may have been corrupted, the provenance pieces embedded into datapoints may not contain all the correct bits of the embedded provenance mark. However, due to the construction of the provenance pieces, it is likely that some information about provenance mark $\mathrm{m}$ can be recovered as a best guess. This guess can be checked for correctness using the mark check bits, as described in Section 4.2. 
For presentation purposes, we define a useful function split( $\cdot)$ that takes datapoint d and, based on parameters $L_{s i g}$ and $N_{p p}$, returns information about the significant bits, check bits, and provenance piece retrieved from d. Formally, for datapoint $d$, and parameters $L_{s i g}$ and $N_{p p}$, we define split (d) $=(\mathrm{s}, \mathrm{pc}, \mathrm{mc}, \mathrm{pp}, k)$ where

$-\mathrm{s}=\mathrm{d}_{0} \ldots \mathrm{d}_{L_{s i g}-1} ;$ and

$-\mathrm{pc}=\mathrm{d}_{L_{s i g}}$ and

$-\mathrm{mc}=\mathrm{d}_{L_{s i g}+1} ;$ and

$-\mathrm{pp}=\mathrm{d}_{L_{s i g}+2} \ldots \mathrm{d}_{|\mathrm{d}|-1} ;$ and

$-k=\operatorname{hash}\left(N_{p p}, \mathrm{~s}\right)$.

Assuming that the datapoint has not been corrupted and $L_{s i g}$ and $N_{p p}$ were the parameters used in the embedding process, then $s$ is the significant bits of $d, p c$ and $\mathrm{mc}$ are the parameter check bit and mark check bit respectively, $\mathrm{pp}$ is the provenance piece that was embedded into the datapoint, and $k$ indicates which provenance piece was chosen for this datapoint. If the datapoint is corrupted, then one or more bits may be incorrect.

However, we provide redundancy in the encoding of the provenance mark in two ways. First, each datapoint contains a provenance piece; even if some datapoints are corrupted, there are likely to be other datapoints that contain the same provenance piece, possibly uncorrupted. Second, the encoding parameter $N_{p p}$ can be set so as to ensure that each bit of the provenance mark appears in more than one distinct provenance piece; if some of the less significant bits of a provenance piece are corrupted due to rounding or truncation, those bits will appear in another provenance piece in a more significant position, making the retrieval process more robust to corruptions such as rounding or truncation. For example, in Figure 2, the first bit of the provenance mark appears in provenance piece $\mathrm{pp}^{3}$ in the fourth least-significant position, and in provenance piece $\mathrm{pp}^{0}$ in the most significant position.

The suggestion for bit $i$ of the provenance mark of datapoint $d$ is the information that datapoint d contains about the $i$ th bit of the provenance mark. Specifically, suggest $(\mathrm{d}, i)$ is either $*$ (if $\mathrm{d}$ contains no information about the $i$ th bit of the provenance mark) or a pair ( $b, c)$, where $b$ is the bit ( 0 or 1 ) that $d$ suggests for the $i$ th bit of the provenance mark, and $c \in \mathbb{N}$ is the confidence of that suggestion. Formally, we define:

$$
\operatorname{suggest}(\mathrm{d}, i)= \begin{cases}\left(\mathrm{pp}_{j}, j\right) & \text { if } j+k \bmod L_{m}=i \text { and } 0 \leq j<|\mathrm{pp}| \\ * & \text { otherwise }\end{cases}
$$

where $(\mathrm{s}, \mathrm{pc}, \mathrm{mc}, \mathrm{pp}, k)=\operatorname{split}(\mathrm{d})$.

The confidence of suggestions is a natural number, where higher numbers indicate less confidence. We use the index within the provenance piece at which the $i$ th bit of the provenance mark occurs. Thus, the most confident suggestion is one where the $i$ th bit of the provenance mark appears in the most-significant position of the provenance piece. This reflects our corruption model, where less-significant bits of a datapoint are more likely to be corrupted than more-significant bits.

For example, if datapoint $d$ is uncorrupted and contains provenance piece $\mathrm{pp}^{2}$ from Figure 2, then suggest $(\mathrm{d}, i)=*$ for $0 \leq i \leq 7$ (since $\mathrm{pp}^{2}$ contains no information about the first 8 bits of the provenance mark $)$, and $\operatorname{suggest}(\mathrm{d}, 10)=\left(\mathrm{pp}_{2}^{2}, 2\right)=(0,2)$.

We lift the definition of suggest $(\mathrm{d}, i)$ from datapoints to datasets: $\operatorname{suggest}(\mathcal{D S}, i)$ is a list of suggestions for bit $i$ of the provenance mark, derived from the datapoints of dataset $\mathcal{D S}$. We ignore datapoints d that contain no information about the $i$ th bit of the provenance mark (i.e., we ignore datapoints d such that $\operatorname{suggest}(\mathrm{d}, i)=*$ ). Formally, we define $\operatorname{suggest}(\mathcal{D S}, i)$ as follows.

$$
\operatorname{suggest}(\mathcal{D S}, i)=\{\operatorname{suggest}(\mathrm{d}, i) \mid \mathrm{d} \in \mathcal{D} \mathcal{S} \wedge \text { suggest }(\mathrm{d}, i) \neq *\} \text {. }
$$


Given suggest $(\mathcal{D S}, i)$, there are many ways to compute the "best guess" for the $i$ th bit of the provenance mark. One possibility is that we select the bit that the majority of suggestions propose, regardless of the confidence of any suggestion. We call this allVote defined as follows (we use $L$ to range over lists of suggestions of the form (b,c), and round $(\cdot)$ rounds real numbers to the nearest integer):

$$
\operatorname{allVote}(L)=\operatorname{round}\left(\frac{\sum_{(\mathrm{b}, c) \in L} \mathbf{b}}{|L|}\right)
$$

Another possibility is that we choose the bit that the majority of the most confident suggestions propose. Function bestVote considers only the most confident suggestions.

$$
\begin{aligned}
\text { bestVote }(L)= & \text { let } L^{\prime}=\left\{(\mathrm{b}, c) \mid(\mathrm{b}, c) \in L \wedge c=\min \left\{c^{\prime} \mid\left(\mathrm{b}^{\prime}, c^{\prime}\right) \in L\right\}\right\} \\
& \text { in round }\left(\frac{\sum_{(\mathrm{b}, c) \in L^{\prime}} \mathrm{b}}{\left|L^{\prime}\right|}\right)
\end{aligned}
$$

Other functions are possible, such as weighting the vote $b$ from suggestion $(b, c)$ based on the confidence $c$-more confident suggestions receive greater weight.

Now, given some function $f$ (such as allVote or bestVote) for computing a bit from a list of suggestions, we can compute a "best guess" $\mathrm{m}$ at a provenance mark from a dataset. The $i$ th bit of the best guess $m$ is computed as

$$
\mathrm{m}_{i}=f(\operatorname{suggest}(\mathcal{D S}, i)) \text {. }
$$

Given thusly computed best guess $m$, we can verify whether it is the originally embedded provenance mark via one-bit checking as described in Section 4.2. The construction of the best guess is robust to many transformations of the dataset; Section 7 presents related analysis in detail.

However, if the dataset is too corrupted, the best guess may be incorrect. If the mark check bits of the dataset are also severely corrupted, then there is insufficient information in the dataset to determine if we have recovered the correct provenance mark. But if the mark check bits are mostly uncorrupted, then we can search for the correct provenance mark, using mc-consistency to determine when we have succeeded.

Directed Search. The space of possible provenance marks is too large to search exhaustively. However, given a best guess for a provenance mark, and some measure of confidence in each bit of that guess, we can direct the search of possible provenance marks so that we check more likely marks first. This allows allocation of a budget for searching, with the budget being used to check the best candidates first.

We assume that we have a best guess $\mathrm{m}$ for the provenance mark, and for each $i$, confidence $c_{i}$ in the $i$ th bit of $\mathrm{m}$. Confidence $c_{i}$ may be derived from the suggestions of datapoints, for example, using the average of confidences of suggestions used to compute the $i$ th bit of $\mathrm{m}$. However, other measures of confidence are possible, such as using the entropy of the suggestions for the $i$ th bit. For example, when using allVote to compute the $i$ th bit of the provenance mark, if $\frac{\sum_{(b, c) \in L} \text { b }}{|L|}$ is close to 0.5 , then there is much entropy in the suggestions for bit $i$, and as a result, there should be low confidence for that bit.

Let $i_{1}, \ldots, i_{L_{m}}$ be a permutation of $1 . . L_{m}$ such that $c_{i_{1}} \geq c_{i_{2}} \geq \cdots \geq c_{i_{L_{m}}}$. That is, $i_{1}, \ldots, i_{L_{m}}$ orders the $L_{m}$ bits of the provenance mark guess $\mathrm{m}$ by increasing confidence, with $i_{1}$ being the index of the bit that we have the least confidence in, and $i_{L_{m}}$ being the index of the bit that we have the most confidence in. The recursive algorithm sketched in Figure 3 checks possible provenance marks in decreasing order of confidence, starting with a call $\operatorname{search}\left(L_{m}, \mathrm{~m}\right)$, where $\mathrm{m}$ is the best guess at the provenance mark. Note that $m$ is the first provenance mark checked, and subsequent possible provenance marks are obtained by varying the bits in which we have the least 


$$
\begin{aligned}
& \operatorname{search}(n, \mathrm{~m}): \\
& \text { if } n=0 \text { then } \\
& \text { check possible provenance mark } \mathrm{m} \\
& \text { else } \\
& \quad \text { search }(n-1, \mathrm{~m}) \\
& \quad \text { flip bit } i_{n} \text { of } \mathrm{m} \\
& \quad \operatorname{search}(n-1, \mathrm{~m})
\end{aligned}
$$

Fig. 3. Directed search algorithm.

confidence. An implementation could stop once the mc-consistency score of a possible provenance mark is above a certain threshold. Alternatively, given a search bound $s b$, it could consider only the first $s b$ possible provenance marks and choose the best provenance mark of those considered. For example, by calling $\operatorname{search}(10, \mathrm{~m})$ only the best $2^{10}$ possible provenance marks will be considered, equivalent to $s b=1024$.

Section 7 discusses the robustness of the retrieval process, and the efficacy of directed search.

\section{EXTENSION TO VARIOUS DATATYPES AND LOW-ENTROPY DATASETS}

Our presentation has focused on embedding and recovery techniques for watermarking collections of bit vectors, with an underlying assumption that lower order bits correspond to less significant data. However, in practice, datasets needing marking may not conform to these assumptions. Here we consider extensions of our framework that can accommodate datasets that may be encountered in the wild.

One precondition for our encoding is a certain level of entropy in data, since significant bits in datapoints serve as provenance piece indices. Insufficient entropy will result in an insufficient distribution of provenance pieces in the dataset. However, we note that surprisingly little entropy is needed to support statistically reliable recovery of a mark; as demonstrated in Section 7 our encoding is reasonably robust for datasets with as few as 50-100 unique elements. Datasets with fewer unique values are uncommon and when they do arise, it is typically when measuring small, discrete quantities. These are a poor fit for our scheme, since encoding in the low order bits of an integral data representation would result in unacceptably large changes in value. On the other hand, increasing entropy in a dataset is always possible, either by randomly manipulating low order bits in datapoints, or by padding real-numbered datapoints with additional, randomized lower order bits. However, this sort of manipulation is so closely tied to the needs and tolerances of data users that we consider such strategies highly application specific and thus out of scope for this presentation.

Real datasets will also employ different representation types of data. For example, geographic coordinates are common in environmental datasets, and are fundamentally different from integral values in their representation. This matter is more readily considered here, since in particular we can develop a general terminology and method for dealing with different data types. Our approach is to introduce a notion of data preserving, bidirectional transformation between representation types and bit vectors. This allows us to retain the previously described method for directly embedding and recovering marks from sets of bit vectors, and providing a technical framework in which to evaluate the effect of applying provenance marks.

We start by formalizing our provenance mark encoding functionality in pointwise manner. For the issue under consideration, it is convenient to consider encoding a single datapoint at a time with fixed encoding parameters. 
Definition 6.1. Recall type datapoint populated by bit vectors. Let $P M$ be our provenance mark embedding function defined on single bit vectors with fixed $L_{p p}, L_{s i g}$, and provenance mark $m$ : mark. Hence:

$$
P M \text { : datapoint } \rightarrow \text { datapoint }
$$

This is the point-wise version of encoding function $E$; the formal relation between these functions is $E(\mathcal{D S}, m)=$ map $P M \mathcal{D S}$. An important property of the encoding is that it only manipulates low-order bits, which we characterize as follows.

Proposition 6.2. Let preservedBits $(P M)=\{i \mid(P M(b))[i]=b[i]$ for all $b$ : bitvec $\}$. Then preservedBits $(P M) \supseteq\left\{0 \ldots L_{\text {sig }}-1\right\}$.

Now, we introduce a general notion of representation type $T$, whose members are data points. The following will show how to effectively mark elements of a type $T$ using a provenance mark embedding function $P M$. As we will see, it is sufficient for $T$ to be endowed with an equivalence relation and a distance function. Distance is expressed as a real number, and allows us to measure the perturbation of datapoints under an embedding.

Definition 6.3. We let $T$ range over arbitrary representation types which must admit an equivalence relation $=_{T}$ and a distance function:

$$
\ominus: T \times T \rightarrow \text { real symmetric distance function }
$$

Now we formally characterize the transformation between representation types and bit vectors, which must be data preserving.

Definition 6.4. Given fixed provenance mark function $P M$ and a representation type $T$, an encoding for $T$, denoted $e$, is a pair of functions vectorize $_{T}$ and devectorize ${ }_{T}$ such that:

$$
\text { vectorize }_{T}: T \rightarrow \text { bitvec } \quad \text { devectorize }_{T}: \text { bitvec } \rightarrow T
$$

and these must be inverses, i.e. for any $p: T$ it must be the case that:

$$
\text { devectorize }_{T}\left(\text { vectorize }_{T}(p)\right)=_{T} p
$$

We will write $e(p)$ to denote devectorize $_{T}\left(P M\left(\right.\right.$ vectorize $\left.\left._{T}(p)\right)\right)$ for $p: T$. $^{2}$

Ultimately, we would like to objectively evaluate encoding schemes to determine if they are suitable for specific applications. Intuitively, this is measured in terms of the resulting perturbation thresholds of datasets-less is better. In general, we expect desirable thresholds to be within the margin of error of a given dataset.

Definition 6.5. An encoding e respects a threshold $n$ in $T$ iff $p \ominus_{T} e(p) \leq n$ for all $p: T$.

Now, some examples. We consider likely common representation types: two's complement integers, fixed-point numbers, IEEE floating points, and geographic coordinates. For both two's complement and IEEE floating point values, transformation is trivial since both representation types are already bit vectors with less significant bits in lower order positions.

Example 6.6 (Two's complement encoding). Take $T$ to be a two's complement bit vector representation of integer values, with maximal bit vector length $n$; we call this type $2 s$. We define vectorize $e_{2 s}$ and devectorize $e_{2 s}$ to be the identity function, and $=_{2 s}$ and $\ominus_{2 s}$ to be ordinary two's complement equality and absolute difference respectively, with results interpreted as real numbers. Suppose that the provenance mark embedding function $P M$ manipulates the $L_{m d}$ low-order bits. Then encoding $e$ respects a threshold $2^{L_{m d}}-1$, since $p \ominus_{2 s} e(p) \leq 2^{L_{m d}}-1$ for all two's complement numbers $p$. 
Example 6.7 (Fixed-point encoding). Take $T$ to be the set of real numbers that, when represented in base-2, have at most $k$ bits after the radix point. Intuitively, by multiplying each datapoint by $2^{k}$, we obtain an integer value, and can use the two's complement encoding defined above. Specifically, we can define vectorize $_{T}$ and devectorize $_{T}$ as follows.

$$
\begin{aligned}
\text { vectorize }_{T}(p) & =\text { vectorize }_{2 s}\left(p \times 2^{k}\right) \\
\text { devectorize }_{T}(v) & =\text { devectorize }_{2 s}(v) \div 2^{k}
\end{aligned}
$$

If we suppose that the provenance mark embedding function $P M$ manipulates the $L_{m d}$ low-order bits then encoding $e$ respects a threshold $2^{L_{m d}-k}-2^{-k}$, which follows immediately for the threshold for the two's complement encoding.

The fixed-point encoding works well if all datapoints in the dataset have approximately the same magnitude. However, if the datapoints can vary in magnitude, then a fixed-point encoding is typically not suitable. This is because the noise added to datapoints to encode provenance information is of the same magnitude for all datapoints, and thus the relative change needed to encode provenance information may be vastly different for different datapoints. This issue can be avoided by using a floating-point encoding to encode numbers of varying magnitude.

Example 6.8 (IEEE floating point encoding). Take $T$ to be the set of real numbers encodable as IEEE binary64 "double precision" floating point numbers; we call this type double. We define vectorize $e_{\text {double }}$ and devectorize double $_{\text {to }}$ to the standard IEEE binary64 encoding and interpretation. Furthermore, we take $=_{\text {double }}$ and $\ominus_{\text {double }}$ to be ordinary IEEE binary64 equality and absolute difference respectively, with results interpreted as real numbers.

In the standard IEEE binary64 encoding, 64 bits are used to represent numbers, with the left-most bit indicating sign $s$ (either 1 or 0 ), the next 11 bits indicating the exponent $e$ (covering all integer values between -1023 and 1023 inclusive) and the remaining 52 bits indicating the mantissa man. Thus, the number represented is $(-1)^{s} \times \operatorname{man} \times 2^{e}$ (with a few exceptions for encoding $\pm 0, \pm \infty$, and not-a-numbers).

Restricting attention to a well-behaved subset of $T$ yields stronger properties. Let $T^{\prime} \subseteq T$ be the set of non-zero, normal numbers. This is all positive and negative doubles with exponent $e$ greater than -1023 and excludes smaller subnormal numbers, \pm 0 , $\pm \infty$, and not-a-number codes. Call the corresponding representation type normdouble. Here we take $a \ominus_{\text {normdouble }} b=\max (a / b, b / a)$. Assuming $L_{\text {sig }}>12$ (i.e., $L_{\text {sig }}$ encompasses the sign bit and all exponent bits), applying a provenance mark does not change a number's sign or exponents bits, and we can calculate that that this encoding respects threshold $1+2^{13-L_{s i g}}$ in $T^{\prime}$.

More interesting is the example of GPS coordinates, which are typically represented as pairs of floating point numbers. Perturbation distance here is measured as the geographic distance between pre- and post-embedding datapoints. Noting that each component of the coordinate pair is equally significant, a successful strategy is to interleave the coordinate pair in vectorization.

Example 6.9 (GPS coordinates). Take $T$ to be pairs of normal real numbers, representing GPS coordinates in WGS84 format up to 64 bit (double) precision; we call this type $G P S$. We define $=_{G P S}$ to be ordinary equality on pairs, and define $\ominus_{G P S}$ as the physical distance between two points on the Earth, calculated using the haversine formula. The result of vectorize $e_{G P S}$ is a 128 bit vector obtained by interleaving the elements of each coordinate pair represented as binary64 values. That is, the least significant bits of the latitude and longitude elements becomes the 0th and 1st bits in the bit vector respectively, the second least significant bits the 2nd and 3rd elements, etc. 
We just take devectorize $_{G P S}$ to be the inverse of this. Modifying the real-number bounds to account for the fact the latitude and longitude each have half as many bits flipped as a single real number, allows us to compute that this encoding satisfies threshold

$$
R \arcsin \sqrt{\sin ^{2} \frac{k \pi}{2}+\sin ^{2} 2 k \pi}
$$

where $R$ is the radius of the Earth and $k=2^{\left(13-L_{s i g} / 2\right)}$. Note that, assuming a constant amount of metadata, $L_{\text {sig }}$ will be more than twice a large for a GPS coordinate-a pair of doubles-as for a single double. As a point of references, if we have 12 bits of metadata then $L_{\text {sig }}=128-12=116$ and the encoding respects a threshold of $2.9 \times 10^{-7}$ meters, or 0.29 micrometers. Section 7.2 evaluates this techinqique in practice.

The essential characteristic of the above transformations from representation types to bit vectors is that less significant data in the representation type end up as less significant bits in the resulting bit vector. Since our encoding modifies the less significant bits of bit vectors, this scheme tends to modify less significant data in the representation type. We therefore propose the following property as a heuristic for creating encoding schemes that are likely to respect low thresholds.

PROPERTY 6.1. Given an encoding $e=\left(\right.$ vectorize $_{T}$, devectorize $\left.{ }_{T}\right)$ for a representation type $T$, we say that e is significant-bit order preserving iff for all $b$ : bitvec we have:

$$
\begin{aligned}
& j<L_{\text {sig } \text { and } j<k \Longrightarrow} \Longrightarrow \\
& \text { devectorize }_{T}\left(\text { flip }_{j} b\right) \ominus \text { devectorize }_{T}(b) \leq \\
& \text { devectorize }_{T}(\text { flip } k b) \ominus \text { devectorize }_{T}(b)
\end{aligned}
$$

where flip i b logically negates b's ith bit.

Finally, when a datatype's vectorized representation contains more than than 64 bits, we may choose to apply the provenance mark encoding and retrieval functions to its least significant 64 bits only. This short-encoding technique does not effect mark perceptibility. Short encoding is poorly suited for datasets whose least significant 64 bits have substantially lower entropy (fewer unique values) than the dataset as a whole. Section 7.2 describes an experiment using short encodings.

\section{EVALUATION AND ANALYSIS}

We have developed prototype tools for encoding provenance marks into datasets, and for performing one-bit and blind mark checking. The tools are implemented in approximately 1,300 lines of non-comment, non-blank lines of Perl code. We have also developed tools that corrupt datasets by rounding, truncating, and sampling datapoints. In this section we empirically evaluate the effectiveness of our techniques using the prototype tools on artificially generated and real datasets. In addition, we consider analytically how our encoding techniques affect statistical properties of datasets.

To ensure consistency of our system's performance on real (not synthesized) datasets and different datatypes, we also considering an encoding of an existing GPS dataset in Section 7.2. Practical experience with encoding of another real dataset is considered in Section 8.

\subsection{Mark checking for synthetic data}

Retrieving encoding parameters. Recall from Section 5 that the first step in both one-bit and blind checking is to retrieve the encoding parameters $L_{s i g}$ and $N_{p p}$ by computing the pc-consistency score of all possible parameters, and selecting the candidate with the greatest score. For an uncorrupted annotated dataset, the correct encoding parameters yield a pc-consistency of 1 , and all other candidates will have a pc-consistency score of approximately $\frac{1}{2}$. 


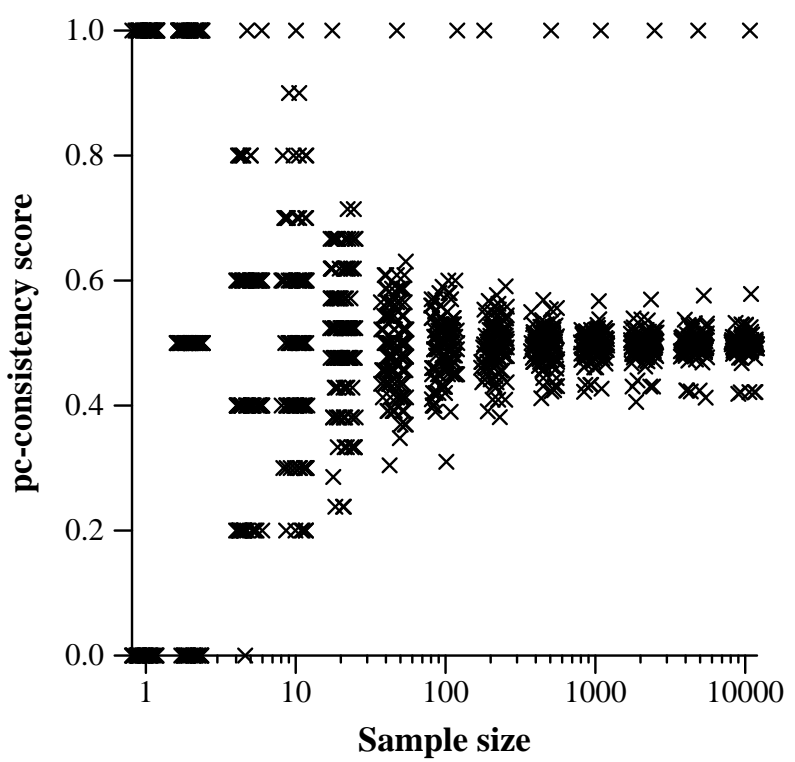

Fig. 4. pc-consistency scores vs. sample size. Each thick column represents pc-consistency scores from a single run of the parameter recovery algorithm.

For corrupted datasets, the correct encoding parameters may have a pc-consistency score less than 1 . Retrieving these parameters is still possible if there exists a significant gap between the greatest and second-greatest pc-consistency scores, which correspond to the correct and best-looking incorrect answer respectively. If the encoding parameters cannot be retrieved, then neither one-bit nor blind checking can be performed.

The retrieval algorithm examines only the significant bits and the parameter check bit of a datapoint; it does not examine the other $L_{m d}-1$ bits of metadata. Thus truncation and bit flips do not effect parameter recovery so long as the corruption is limited to low-order bits. Furthermore, pc-consistency scoring is unaffected by dataset reordering. Below we consider how sampling and rounding affect parameter retrieval.

Corruption via sampling. Figure 4 shows the pc-consistency scores for all non-trivial encoding parameter candidates for annotated datasets of various sizes. Samples were drawn from a synthetic dataset with 10,000 datapoints generated by choosing elements uniformly at random, with replacement, from the set $\left\{1,2, \ldots, 5 \times 10^{6}\right\}$. We assume that there are 13 significant bits $\left(L_{s i g}=13\right)$, and, since the synthetic data can be represented in 23 bits, there are 10 least significant bits that we can use to encode metadata $\left(L_{m d}=10\right)$. A 32-bit provenance mark $\left(L_{m}=32\right)$ was encoded in the dataset by replacing the 10 least significant bits; there were 8 distinct provenance pieces $\left(N_{p p}=8\right)$, and the length of each provenance piece was $8\left(L_{p p}=L_{m d}-2=8\right)$. Unless otherwise stated, all experiments in this section used the same parameters. When retrieving the encoding parameters, degenerate encoding parameters (with $L_{\text {sig }} \leq 2$ ) were ignored.

In all cases, the correct parameters have a pc-consistency score of 1 . For sufficiently large samples (say, at least 50), the pc-consistency scores of incorrect parameters cluster around $\frac{1}{2}$, since incorrect parameters are consistent with a given parameter check bit half the time, and thus the pc-consistency scores of incorrect parameters are bi- 


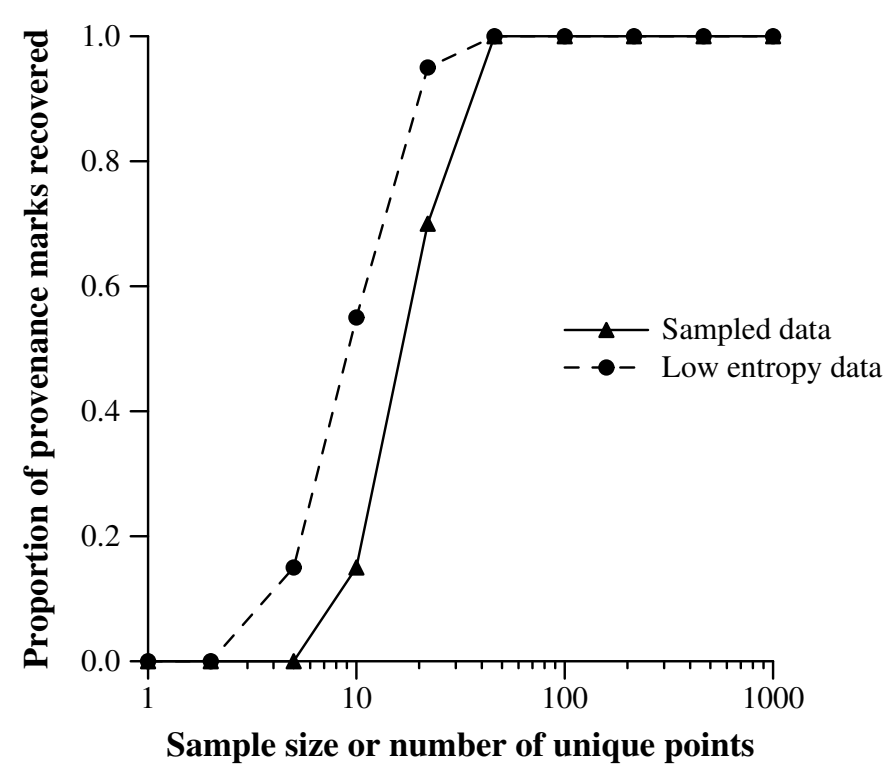

Fig. 5. Fraction of provenance marks recovered from a data set vs. number of unique datapoints. The solid series describes data sets with $k$ elements and the dashed series describes data sets with 1,000 elements, $k$-many of which are unique. Prior to decoding, low-order bits are flipped with low probability. The difference between sampled and low-entropy behavior appears to vanish if the encoded data is not corrupted.

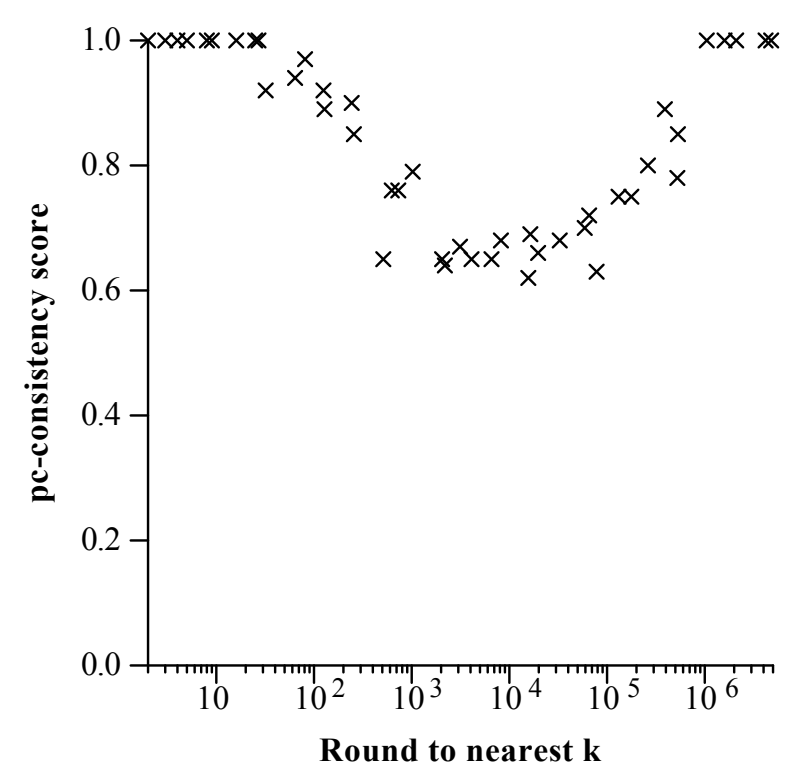

Fig. 6. Max pc-consistency scores vs. degree of rounding. 
nomially distributed. Thus, in a dataset with $n$ distinct values, the probability of an incorrect parameter have a pc-consistency score of 1 is approximately $2^{-n}$. For small sized samples, there is a much greater chance of an incorrect parameter having a high pc-consistency score.

Thus, the correct encoding parameters can be retrieved with high probability given a sufficiently large sample of an otherwise uncorrupted annotated dataset, say at least 50 datapoints. This result is independent of the length of the provenance mark $L_{m}$, length of the metadata $L_{m d}$, and number of provenance pieces $N_{p p}$.

Figure 5 shows the proportion of provenance marks recovered when computed from a sampled data set. Consistent with Figure 4, a high likelihood of successful provenance mark recovery was observed for samples with about 50 elements.

Effect of low-entropy data. We consider a dataset with many identical values to be a low-entropy dataset. Such a dataset may occur when, for example, a sensor measures a slowly changing quantity. Provence mark recovery for a data set with many samples but only $k$ unique elements behaves similarly to recovery for a data set with $k$-many elements total, all of which are unique. Figure 5 shows that a low entropy data set can be more resilient to random corruption than a small sample of a data set. A point is corrupted by flipping each of its 4 least significant bits with probability 0.2 .

Corruption via rounding. Figure 6 shows maximum pc-consistency scores computed when decoding a dataset corrupted by rounding datapoints to the multiples of $k$, for varying values of $k$. The dataset contained 10,000 datapoints, but the pc-consistency scores were calculated using a randomly chosen 100 element sample.

As we round by larger quantities, top scores fall and it becomes harder to distinguish the correct encoding parameters from the incorrect parameters. Rounding to the nearest multiple of $k$ for $2 \leq k \leq 128$ leaves top pc-consistency scores far above those expected for arbitrary parameters, thus allowing the successful retrieval of the encoding parameters. At first, large values of $k$ yield substantially lower top pc-consistency scores. However once rounding has eliminated enough significant bits, it becomes easy to overfit during parameter recovery.

The value of $k$ for which it becomes difficult to determine the correct encoding parameters is independent of the length of provenance mark $L_{m}$ and number of provenance pieces $N_{p p}$. It is however dependent on the length of the metadata $L_{m d}$ : using more bits for metadata provides robustness for larger values of $k$.

One-bit checking. One-bit checking evaluates the mc-consistency score of a single provenance mark. A sufficiently high mc-consistency score indicates that it is likely that the same provenance mark was embedded in the dataset.

Since one-bit checking uses just the mark check bit, and does not use the $L_{p p}=$ $L_{m d}-2$ bits of the provenance piece, its robustness with respect to various corruption is very similar to that of recovering the encoding parameters, which uses the parameter check bit. It is unaffected by truncation or bit-flipping that affect only the $L_{m d}-2$ least-significant bits of datapoints. It is also unaffected by reordering of datapoints. The effects of sampling and rounding on one-bit checking are the similar to their effect on parameter recovery.

What mc-consistency score indicates that we have the same provenance mark that was used during encoding? The acceptance threshold for mc-consistency scoresespecially in view of potential corruption-is best determined empirically. In our experience, 0.85 is a conservative threshold; given a sufficiently large dataset (containing, say, 100 distinct values), the probability that an incorrect provenance mark will have a mc-consistency score of 0.85 or higher is approximately $2.41 \times 10^{-13}$-about two chances in 10 trillion. Intuitively, this is because the mc-consistency scores of incorrect provenance marks are binomially distributed. Figure 7 demonstrates this with a histogram 


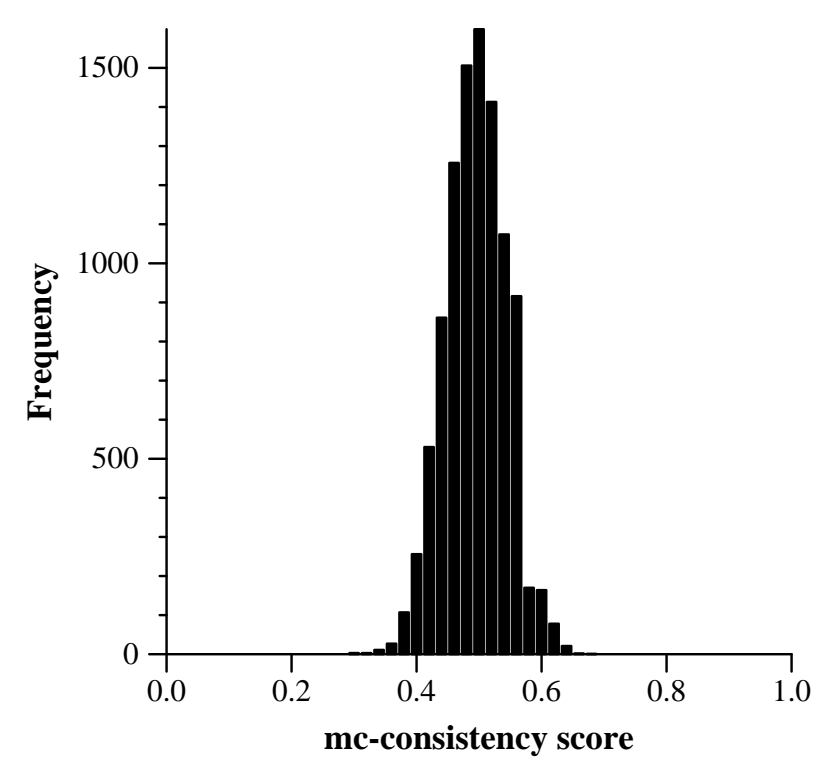

Fig. 7. mc-consistency scores of incorrect provenance marks.

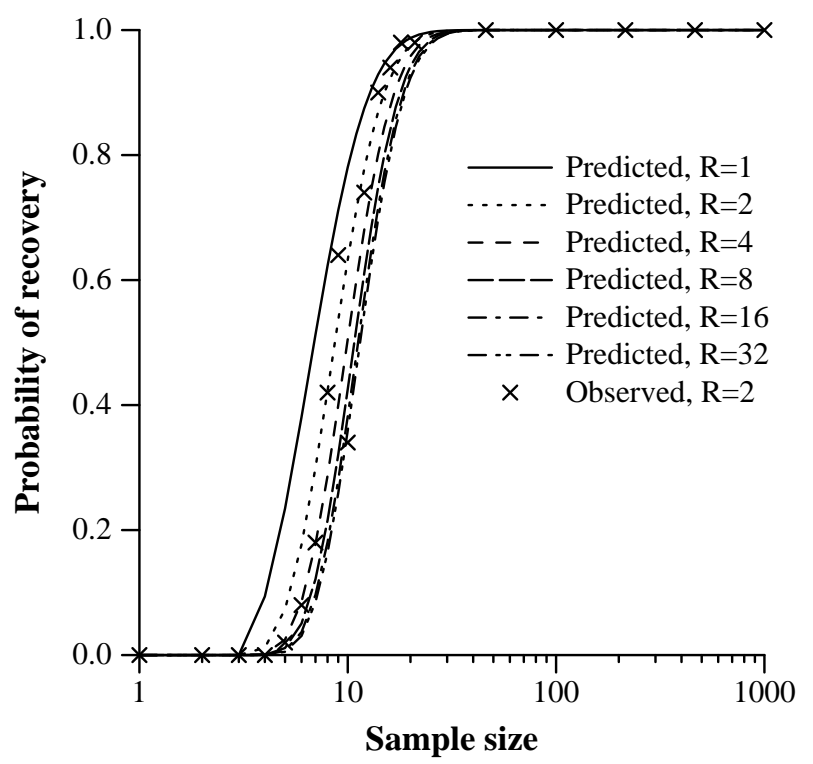

Fig. 8. Predicted and observed probability of recovering provenance mark. 
of mc-consistency scores for about 10,000 incorrect provenance marks, in a dataset of 100 datapoints.

Blind checking. Blind checking attempts to retrieve a provenance mark from a corrupted annotated dataset knowing only the length of the mark. It uses a heuristic to generate one or more guesses, and uses one-bit checking as a subroutine to evaluate the guesses. Blind checking is not affected by reordering of datapoints. It is affected by sampling, since too few datapoints may not contain all bits of the encoded provenance mark. Blind checking can be affected by bit flips, truncation, and rounding; however, the encoding scheme is designed to be robust to corruption in lower order bits, and truncation and rounding are more likely to corrupt lower order bits.

Effect of redundancy and sampling. A single bit of the provenance mark may occur in many provenance pieces. We write $R=k$ to indicate an annotated dataset has redundancy $k$ - that is, each bit of the provenance mark appears in $k$ provenance pieces. As shown in Figure 2, encoding a provenance mark of length 16 using $N_{p p}=4$ and $L_{p p}=8$ results in each bit of the provenance appearing in two distinct provenance pieces: $R=2$. Increasing redundancy substantially increases likelihood that decoding will succeed in the presence of corruption of lower-order bits. However, more redundancy also increases the probability that a small sample won't contain all bits of a provenance mark.

The probability of recovering a provenance mark from an (uncorrupted) annotated dataset of size $|D|$, with a given redundancy $R$, can be calculated analytically. Each datapoint of the annotated dataset contains one of the $N_{p p}$ distinct provenance pieces, chosen uniformly at random by the encoding mechanism. Thus, there are $\left(N_{p p}\right)^{|D|}$ equally probable ways of choosing (with replacement) $|D|$ provenance pieces from the $N_{p p}$ distinct provenance pieces. Given $|D|$ provenance pieces, the provenance mark can be recovered if every bit of the provenance mark occurs in at least one of the $|D|$ provenance pieces. We can count the number of ways that $|D|$ provenance pieces can be chosen such that the provenance mark can be recovered. The combinatorial argument is described in detail in the accompanying technical report [Chong et al. 2014]. Figure 8 presents the predicted probability of recovering a provenance mark from annotated datasets of various size (with $N_{p p}=8$ ). Here, recovery means that the sample contains datapoints with enough provenance pieces so that every bit of the provenance mark appears in at least one provenance piece. Other than sampling, the annotated dataset is not corrupted (i.e., no bit-flipping, rounding, etc.). Note that the probabilities for recovery depend only on the redundancy and sample size, and are independent of provenance mark length $L_{m}$, and provenance piece length $L_{p p}$; however, not all redundancies are possible with given encoding parameters. For example, $R$ cannot be greater than $L_{p p}$.

Figure 8 demonstrates that the probability of blind mark recovery undergoes a "phase transition" around sample size 10. For smaller samples, there are not enough distinct provenance pieces present to recover all the bits of the provenance mark; for larger samples, recovery is very likely.

Also plotted on Figure 8 are observed recovery rates for a series of experiments using $L_{m}=32, L_{p p}=8$ and $N_{p p}=8$ (which implies $R=2$ ). Taking samples of various sizes from a large uncorrupted annotated dataset, we measured how often the sample contained sufficient information to reconstruct all bits of the provenance mark (without any search). The observed success rate matches the predicted success rate well.

Corruption via truncation and rounding. Unlike one-bit checking and parameter retrieval, blind checking uses low-order bits to identify a best-guess provenance mark; corruption via truncation or rounding can impede blind checking. 
Truncation may occur in one of two ways. Under zeroing truncation, low bits of a datapoint are overwritten with zeros, losing precision but maintaining the datapoint's order of magnitude. For instance applying three bits of zeroing truncation to 53 (binary 110101 ) yields 48 (binary 110000). In contrast shifting truncation simply removes the truncated bits, reducing values by factors of two. For instance, three bits of shifting truncation takes 53 to 6 (binary 110).

Figure 10 shows the effect of zeroing truncation of blind checking. All datapoints of an annotated dataset had the $n$ least-significant bits set to zero; blind and directed search performed, with a search bound of $2^{16}=65,536$. That is, at most $2^{16}$ provenance marks were considered. The $\mathrm{x}$-axis indicates $n$, the number of least-significant bits truncated from each datapoint; the y-axis shows how many provenance marks were considered before the provenance mark with the highest mc-consistency score was found (which may not be the correct mark). Ten trials were performed for each possible combination of $R \in\{1,2,4,8\}$ and $0 \leq n \leq 9$, and the mean is plotted.

The graph indicates that we "fall off a cliff". Blind checking can tolerate some amount of zeroing truncation very well, and is able to retrieve the provenance mark without search (rank $=1$ ). However at some point, too many bits have been truncated, and we are unable to recover the provenance mark. Higher redundancy results in more robustness to truncation. The correct provenance mark, if not found at rank 1 , was typically not in the first $2^{16}$ provenance marks considered.

Comparing Figures 10 and 11 indicates zeroing and shifting truncation have similar effects. Furthermore, our experiments indicate that the effects of rounding are also similar.

\subsection{Mark encoding and retrieval for GPS data}

Section 6 describes a vectorization scheme for GPS data and introduces a shortencoding technique that adds and retrieves provenance marks using only the low 64 bits of a datapoint. We applied these techniques sets of GPS coordinates used by the Bridger-Teton Avalanche Center ${ }^{2}$ to define avalanche forecast boundaries.

Each of 51 regions is described by 7-178 coordinates. For each region we attempted to encode and decode 10 randomly generated 32-bit provenance marks. Consistent with the analysis above, mark retrieval always succeeds for regions with at least 41 points and succeeds in $63 \%$ of trials for regions with at most 32 points. (No region has $33-41$ points.) Recovery rates are summarized in Figure 9.

\subsection{Perceptibility}

Embedding of a provenance mark into a dataset should not affect the scientific use of the dataset. Our embedding technique is clearly detectable: algorithms such as one-bit checking can distinguish annotated datasets from unannotated datasets. However, we alter only the least significant bits of datapoints-those within the noise of measurement. As such there is little impact on various statistical measures of the dataset and no significant impact on many of the scientific uses of the dataset.

For specific descriptive statistics, we can analytically bound the effect of embedding marks into datasets. Let $L_{m d}$ be the number of bits of metadata (parameter check bit, mark check bit, and provenance piece) that we are adding to each datapoint.

Mean. By adding metadata to a datapoint, the value of a datapoint can change by at most $2^{L_{m d}}-1$. Thus, the mean over the dataset changes by at most $2^{L_{m d}}-1$. However, typically the actual change to the mean would be at least an order of magnitude smaller, since the first two bits of the metadata (the parameter and mark check bits) are uniformly distributed. Additionally, if the lower order bits of the original datasets

\footnotetext{
${ }^{2}$ http://www . jhavalanche.org/index.php
} 


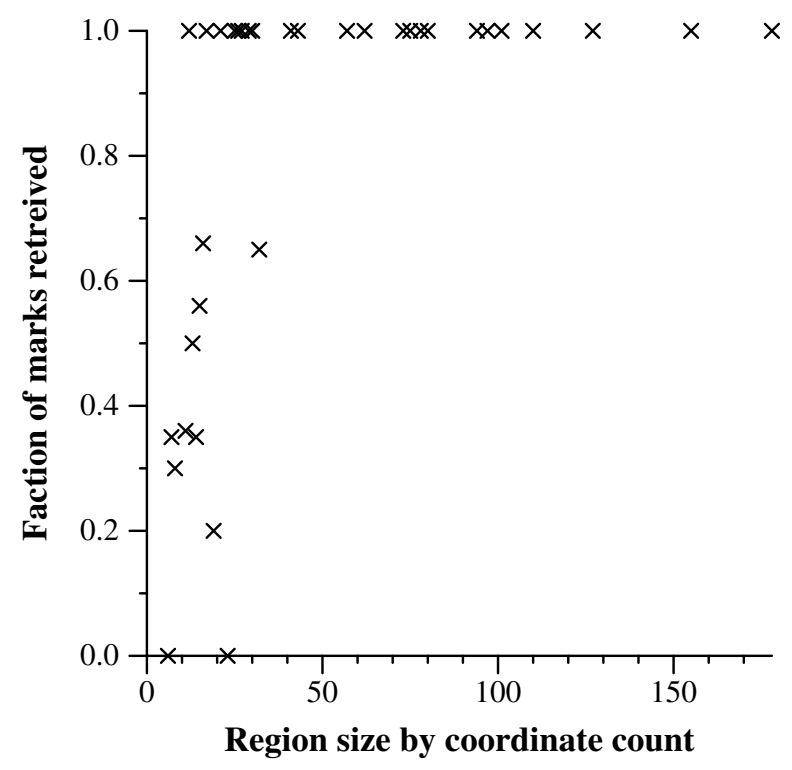

Fig. 9. Fraction of randomly generated provenance marks recovered in regions bounded by varying numbers of GPS coordinates in the Bridger-Teton Avalanche Center dataset. All marks are recovered for regions with at least 41 elements. (Here $L_{m d}=6$.)

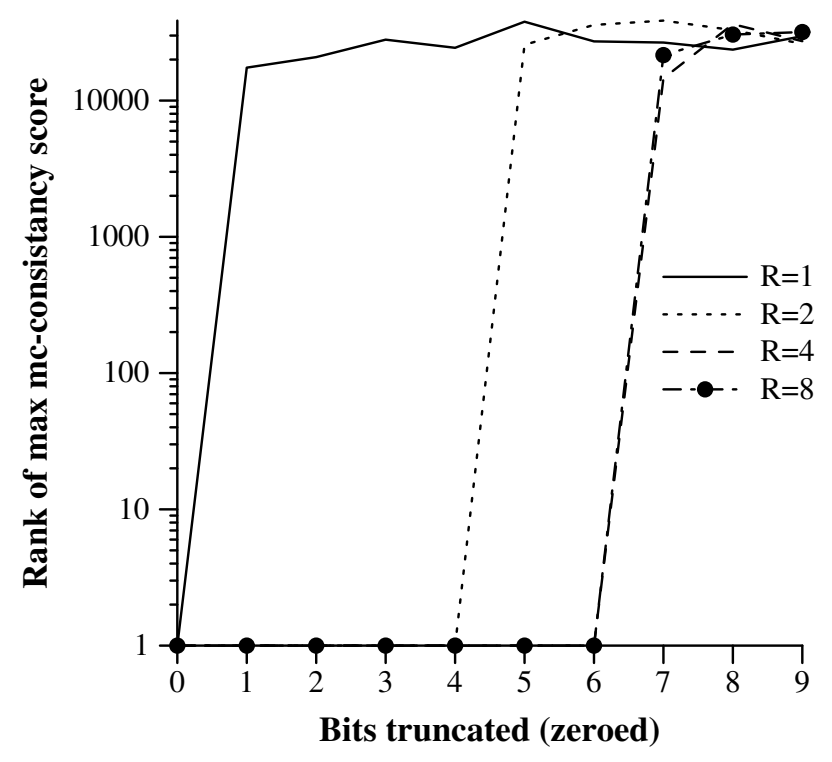

Fig. 10. Rank of max mc-consistency score vs. zeroing truncation to nearest k. Sample contains 100 elements. 


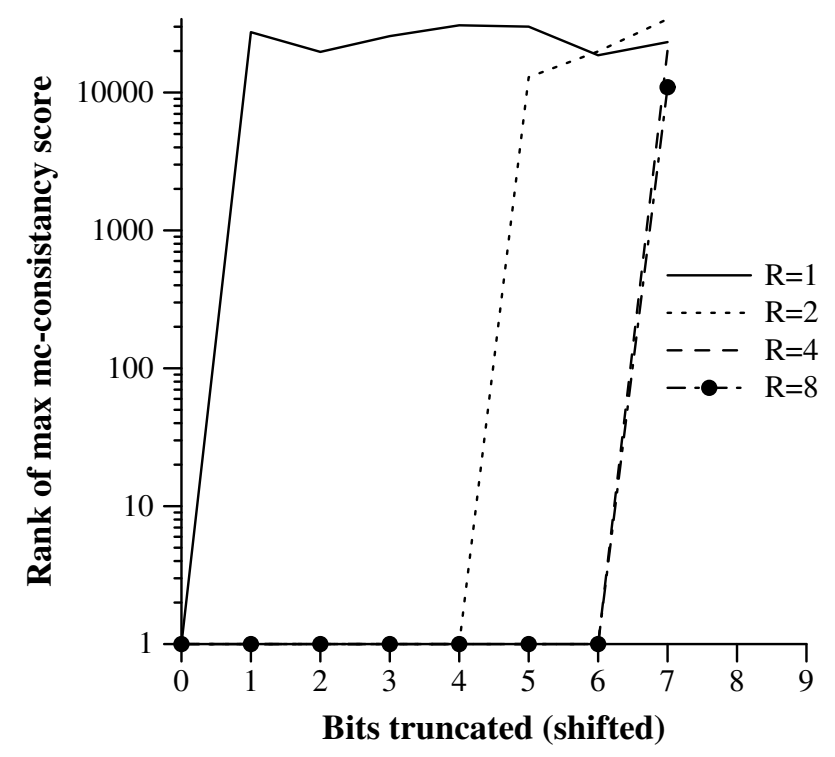

Fig. 11. Rank of max mc-consistency score vs. shifting truncation to nearest k. Sample contains 100 elements. Recovery fails completely when points are shifted by $L_{p p}(=8)$ bits.

are fairly uniformly distributed over the interval $\left[0,2^{L_{m d}}-1\right]$, then the change in the mean will be close to zero.

Variance. We assume that the value of the $L_{m d}$ least significant bits of an unannotated dataset are not correlated with the value of the more significant bits; this is a reasonable assumption since the errors in measurement are greater than $2^{L_{m d}}$.

Let $X$ be the random variable corresponding to the distribution from which the unannotated datapoints are sampled. Let $\epsilon=2^{L_{m d}}$. The following equivalence holds.

$$
X=\epsilon\left\lfloor\frac{X}{\epsilon}\right\rfloor+X \bmod \epsilon
$$

Let $M$ be the random variable corresponding to the distribution from which the metadata is taken, which ranges over the interval $[0, \epsilon-1]$. Note that since the metadata is selected using hash functions, the metadata added to a datapoint is independent of the datapoint.

The annotated dataset is obtained by replacing the last $L_{m d}$ bits of each datapoint with metadata. The annotated dataset is modeled by random variable $A$ where

$$
A=\epsilon\left\lfloor\frac{X}{\epsilon}\right\rfloor+M
$$


The variances of $A$ and $X$ are given by

$$
\begin{gathered}
\operatorname{Var}(X)=\operatorname{Var}\left(\epsilon\left\lfloor\frac{X}{\epsilon}\right\rfloor\right)+\operatorname{Var}(X \bmod \epsilon) \\
+\operatorname{Cov}\left(\epsilon\left\lfloor\frac{X}{\epsilon}\right\rfloor, X \bmod \epsilon\right) \\
\operatorname{Var}(A)=\operatorname{Var}\left(\epsilon\left\lfloor\frac{X}{\epsilon}\right\rfloor\right)+\operatorname{Var}(M)+\operatorname{Cov}\left(\epsilon\left\lfloor\frac{X}{\epsilon}\right\rfloor, M\right) .
\end{gathered}
$$

Distribution $\epsilon\left\lfloor\frac{X}{\epsilon}\right\rfloor$ is independent of $X \bmod \epsilon$ and of $M$, so both covariance terms are 0 . Thus,

$$
\operatorname{Var}(X)-\operatorname{Var}(A)=\operatorname{Var}(X \bmod \epsilon)-\operatorname{Var}(M) .
$$

Both $X \bmod \epsilon$ and $M$ sample $\{0, \ldots, \epsilon-1\}$, so their variances are at most $(\epsilon-1)^{2} / 4$ (see Jacobson [1969]). Thus the worst case change in variance is $\operatorname{Var}(X)-\operatorname{Var}(A)=$ $\pm(\epsilon-1)^{2} / 4$. If both $X$ 's low order bits and distribution $M$ are uniformly random, then $\operatorname{Var}(X)-\operatorname{Var}(A)=0$.

\section{THE TECHNIQUE IN PRACTICE}

In practice, we envision that provenance information will be encoded in data at its source, and online or offline tools will be available to retrieve provenance information from encoded datasets. Since the encoding and decoding algorithms are completely described in this paper, it is straightforward to provide encode and decode utilities. To illustrate and explore possible implementations, we have made a prototype decode utility available online at http://tinyurl.com/q3bddpf. This utility requires that input data is in tab-delimited multi-column format. Encoded data may be either integer or floating point numbers. The utility will decode any provenance mark in the dataset using techniques described in Section 4, Section 5, and Section 6, and then report either that no known mark was successfully retrieved, or report the provenance information associated with the mark.

Our implementation assumes that provenance marks are 32-bit integer identifiers and maintains a database associating those identifiers with provenance information. The decode utility retrieves associated provenance information from the database, and reports it to the user via a webpage redirect. Note the implicit semantics of provenance marks in this implementation, i.e. as database lookup keys. Another appealing option is to interpret provenance marks as URLs directly. However, even URL minimization services such as Bitly and TinyURL have more than 32 bits of entropy in their addresses, and it appears that 64 bit marks would be necessary to interpret marks as (e.g.) Bitly url references. Our core encoding and decoding tools support such longer provenance marks, and updating the web frontend to would be an an easy extension to our system.

As revealed in Section 7, about 50 unique datapoints in a dataset are required to reliably encode and retrieve a provenance mark. From a cursory examination of datasets in [Hubbard Brook Ecosystem Study ], this does not appear to be an unusual or onerous requirement for environmental datasets at least. The dataset we have encoded in our online demo possesses ample entropy of this sort.

Experience with an encoded dataset. We have encoded a 32-bit provenance mark in a publicly available dataset. This is a time-series dataset of snow depth readings from a deployment adjacent to route 395 near Mammoth Lakes, CA. These readings were taken at hourly intervals during the Winter of 2012 . The data is available via an interface available online at: http://tinyurl.com/16a2wx3. To access the encoded data, choose the "encoded data" panel, and select Snow Depth data from any subset of Tow- 
ers 1,2 , and 3 specifying start and end times during the period $1 / 11 / 12-4 / 30 / 12$. The Table output format should be selected, which will enumerate data in a tab delimited multicolumn format. An adequate selection of data (50 datapoints or so) can be copy-pasted directly into the decode tool described above.

Using one data set, Tower 2 snow depth, we examined continuous data windows of different length, and considered how this impacts mark recovery. We attempted to decode a provenance mark for 100 random selected intervals of each duration, and found that mark recovery was reliable for periods of 60 hours, with successful recovery occurring in $98 \%$ of windows. Recovery succeed in all trials with windows of 84 or more hours. The data is summarized below:

\begin{tabular}{|l|ccccccccc|}
\hline Period duration (hours) & 12 & 24 & 36 & 48 & 60 & 72 & 84 & 96 & 108 \\
\hline Marks recovered & $24 \%$ & $59 \%$ & $83 \%$ & $91 \%$ & $98 \%$ & $99 \%$ & $100 \%$ & $100 \%$ & $100 \%$ \\
\hline
\end{tabular}

\section{DEPLOYMENT ISSUES AND FUTURE WORK}

In this paper we develop a foundational theory comprising an abstract notion of "dataset," and provide a prototype implementation. However, our scheme is intended for real-world deployment and this raises a variety of issues. Here we discuss salient ones and how they can be approached in future work.

Social issues. As we have stressed, our scheme is not a security mechanism per se, but rather is intended to (1) support the "fair use" policies typical of publicly available environmental sciences data, and (2) provide a means to mark data with its own metadata. The importance of both fair use policies and metadata in the environmental sciences community is evidenced by online archives such as the aforementioned HBES [Hubbard Brook Ecosystem Study] and the Sagehen Creek Field Station repositories [Sagehen Creek Field Station Data Repository ]. Both incorporate policies expecting that data producers and archivers should be acknowledged in and informed of publications that use their data. These sites also clearly associate metadata with datasets, which is crucial to contextualize environmental data.

Thus, the tools and techniques we propose should be freely available and "open source," and our provenance encoding need not be irreversible in a cryptographic sense; they are intended to support scientists and promote good citizenship. In particular, we envision publicly accessible web-based tools for embedding and retrieving provenance marks. Indeed, probably the most sensitive aspect of our scheme is that it alters datasets themselves. Data producers often feel strongly about the integrity of their data and may look askance at manipulation of their data, arguments about imperceptibility notwithstanding. However, in our scheme it is always the case that unmarked data is available to the data producer and can be privately archived. In the online data repository described in Section 8, we have made both marked and unmarked datasets available through separate interfaces. It would be straightforward for the data producer to rescind public access to the unmarked dataset interface.

The meaning of provenance marks. Intuitively, provenance encoding provides a means to answer the questions "where did this data come from?" and "is this data mine?", given just a dataset. Analogously to typical watermarking schemes, blind checking addresses the former question, while one-bit checking addresses the latter. Which question is more important may determine what meaning is carried by the provenance marks. That is, if checking data ownership is paramount, then it would suffice that each data producer uses a unique provenance mark, and a producer's single mark may be embedded in many datasets. On the other hand, if provenance is the dominant issue, then marks would more appropriately encode or point to the dataset's metadata. For example, a mark could be a url for a webpage containing extensive provenance information for the marked dataset; a level of indirection allows marks to 
be shorter than metadata, allowing a more robust encoding. This is the approach we have used in our prototype online tool as described in Section 8.

Embedding and data lifecycles. A central issue is at what point in the data lifecycle should provenance marks be embedded. In particular, note that raw sensor data is usually obtained as a straight voltage reading or as a ratio to a benchmark voltage. Some formula is then applied to obtain a standard unit measurement based on the calibration of the instrument. Since this transformed reading is typically the data that is actually disseminated, we argue that transformed data in standard units is more appropriate to be marked than raw sensor data. Otherwise, our provenance encoding scheme would have to be robust to arbitrary algebraic transformations, or the retrieval algorithm would need to "know" the precise transformation function, neither of which is realistic.

At what point the embedding occurs is also a function of who intends to mark the data; when data is included as part of a larger repository then both the producer and the archiver may wish to mark it with provenance information. If the latter, the embedding could be performed upon data entry into or retrieval from the repository in a straightforward manner. If the former, it may be desirable to perform the embedding before the data is introduced to the repository, e.g. as part of data collection in an embedded system. Given empirical observations regarding the efficiency of our scheme we believe this is practical. The most computational expensive component of our embedding technique is the computation of a hash; MD5, a commonly-used cryptographic hash function, has been implemented as a MAC algorithm for TinyOS [UbiSec\&Sens Hmac-MD5 Implementation ]. Indeed, since our scheme is not a security mechanism, we do not require our hash to be hard to invert, and could just as well use a block cipher algorithm instead of a hash; implementations of, for example, both AES and Skipjack are available in TinyOS.

Retrieval efficiency. A problem related to retrieval tools is larger datasets. Obviously, the larger the dataset, the longer the retrieval process. Very large datasets could pose a significant problem for our technique unmodified. However, we have observed that our technique is robust to sampling in both our combinatorial analysis and empirical observations. In particular, Figure 8 shows that approximately 100 datapoints with 10 bits of metadata provides high reliability of recovery of a 32-bit provenance mark. Thus, one-bit and blind checking could be implemented efficiently for large datasets by sampling a relatively small subset of datapoints.

\section{RELATED WORK}

The issue of how to represent and manage metadata and provenance in environmental data repositories has received increasing attention as these repositories grow in size and popularity. Previous related work has considered tracking provenance of data as it is republished [Park and Heidemann 2008] and during curation of large-scale, interconnected data repositories [Baker and Yarmey 2009], as well as leveraging provenance information to increase the utility of environmental data [Ledlie et al. 2005]. Research has resulted in online tools for sensor data storage such as SensorBase [Chang et al. 2006] which have even introduced the notion of "slogging", or logging of sensor data via systems that automate metadata annotation and support sharing with the broader community. While this previous work reflects the importance of issues we have addressed in our work, it mainly considers management and dissemination of metadata and provenance annotations, rather than introducing means to directly associate data with its metadata as in our system.

Our provenance encoding technique can be viewed as a new type of digital watermarking [Fridrich and Goljan 1999; Cox and Miller 2002]. A vast amount of research exists in this field, with watermarking techniques proposed for a variety of media; most 
related to our work are techniques for watermarking relational databases [Agrawal and Kiernan 2002; Shehab et al. 2008]. Such relational watermarking techniques tend use primary keys in the same way we use significant bits-as important, unalterable parts of a tuple, but this is not essential and our datasets may be viewed as simple, unary relations. The watermarking literature has also considered the use of watermarks to associate data with its provenance information. Previous work has treated this issue in multimedia DBMS [Chbeir and Gross-Amblard 2006] and in raw video data [Gehani and Lindqvist 2007]. Our work accomplishes similar goals for environmental datasets. However, a key difference with most work on watermarking is that we do not regard the user of the data as an adversary who is attempting to detect or remove the watermark. As such, our technique does not aim to be imperceptible (although we do ensure that embedding does not affect scientific uses of the data), and we do not aim for the metadata to be inalterable. A notable exception is the VEIL system for certifying video provenance [Gehani and Lindqvist 2007], which allows an adversary to remove metadata, but aims to make it difficult for an adversary to introduce false metadata, or to alter data while still associating it with the same metadata.

Watermarking has been used in distributed protocols for WSNs, to support provenance and copyright protection [Harjito et al. 2012] as well as data integrity [Kamel and Juma 2011]. However, the goal of these works have been to support security and copyright protection in potentially hostile network environments, using algorithms integrated with network communication protocols to establish something similar to a secure channel. In contrast, our work is intended to support fair use policies and provenance in data communication between people, and assumes a relatively benign threat model.

Agrawal and Kiernan [2002] introduce techniques similar to ours, in particular, using hashes to identify insignificant bits subject to alternation in the watermarking process. Unlike our work, their technique only supports one-bit checking; because an unknown watermark cannot be recovered from a dataset the technique is not suitable for embedding provenance identifiers. Additionally, their encoding algorithm does not allow the use of multiple low-order bits or redundancy to improve robustness.

The work of Sion et al. [2006] considers watermarking streaming data. Their work is motivated by the desire for a tamper-resistant algorithm, and they assume datasets must be ordered streams. In contrast, our work is non-adversarial and makes no particular assumptions about streaming. Toward tamper-resistance Sion et al. present several watermarking schemes that modify the significant bits of some datapoints, so that removing the watermark fundamentally damages the data and enables detection of the edit. This watermarking technique is robust to a different class of transformations than ours. For example, in Sion's work a contiguous stream segment can be replaced by an average, but datapoints cannot be reordered.

Shehab et al. [2008] make explicit the importance of not alternating most significant bits when adding provenance metadata to a dataset, identifying that preserving MSBs is useful both to maintain data utility and to provide stable input for metadata encoding and decoding algorithms. Shehab's technique works over relational data and defines a tuple's primary key as its MSBs, and a hash of these bits is used to split relations into disjoint partitions, each of which is able to encode a single bit of provenance information. Similarly, we use MSBs to determine a provenance piece associated with a datapoint. However one of our provenance pieces carry multiple bits of metadata, while the entire set of datapoints in one of Shehab's partition may carries at most one bit. Unlike us, Shehab assumes an adversarial setting.

Tracking provenance in curated databases [Buneman et al. 2006] is another related problem of burgeoning interest. Here the issue is keeping track of the provenance of data originating from multiple sources, manually constructed by domain experts. 
But work in this area treats structured data and focuses on the "history" of data and how it is manipulated to obtain the curated database. Somewhat more related is work on tracking provenance in automatically-generated data warehouses [Bhagwat et al. 2005; Tan 2003; Cui and Widom 2001; Lee et al. 1998; Sadri 1998; Widom 2004]. This work mostly focuses on combining provenance annotations on data warehouses in a systematic manner; by contrast, we aim to directly associate provenance information with unstructured data.

\section{CONCLUSION}

In this paper we have a presented a system for generating self-identifying data, which is environmental sciences data marked with its own metadata in an automatically recoverable manner. Our system is intended to support fair-use policies in the environmental sciences community. Our technique for marking data is similar to previous watermarking techniques for other media, in that we define mark embedding and both one-bit and blind retrieval algorithms that are robust to a number of transformations. Since our system is not intended as a security mechanism per se, the transformations we consider are benign and comprise modifications we expect data users to make in the course of normal study, including sampling, reordering, truncation, and rounding of data. We have performed combinatorial and empirical analysis of our system characterizing its robustness in various scenarios and providing insight into its best use. Finally, we built a prototype implementation and applied our technique to time-series data collected in the field.

\section{Acknowledgments}

We thank James Cheney for inviting us to a meeting at the University of Edinburgh where we started this work, and are grateful for travel support from the UK eScience Institute Theme Program on Principles of Provenance. Thanks to Evan Davis-Drennan and Rebecca Norton for their work on implementing the online decoder utility. Thanks also to Jeff Brown of Sagehen Field Station for inspiring the idea, and to the reviewers and shepherds of previous versions of this work for useful feedback. Christian Skalka's work was supported by a grant from the Air Force Office of Scientific Research.

\section{REFERENCES}

AGRAWAL, R. AND KIERNAN, J. 2002. Watermarking relational databases. In Proceedings of the 28th International Conference on Very Large Data Bases. Morgan Kaufmann Publishers, San Francisco, CA, $155-166$.

ARndt, H. K., BAndholtz, T., GÜnther, O., RÜTher, M., AND SchütZ, T. 2000. EML - the Environmental Markup Language. In Workshop Symposium on Integration in Environmental Information Systems. ISESS, $1-9$.

BAKER, K. S. AND YARMeY, L. 2009. Data stewardship: Environmental data curation and a web-ofrepositories. The International Journal of Digital Curation 4, 2, 12-27.

Bhagwat, D., Chiticariu, L., Tan, W. C., And ViJayvargiYa, G. 2005. An annotation management system for relational databases. The VLDB Journal 14, 4, 373-396.

Buneman, P., Chapman, A., AND Cheney, J. 2006. Provenance management in curated databases. In Proceedings of the 2006 ACM SIGMOD International Conference on Management of Data. ACM, New York, NY, USA, 539-550.

Chang, K., YAU, N., HANsen, M., AND Estrin, D. 2006. Sensorbase.org - a centralized repository to slog sensor network data. In Proceedings of the International Conference on Distributed Computing in Sensor Network (DCOSS) / Euro-American Workshop on Middleware for Sensor Networks.

ChBeir, R. AND GRoss-AmblaRd, D. 2006. Multimedia and metadata watermarking driven by application constraints. In 2006 12th International Multi-Media Modeling Conference Proceedings,. IEEE, Washington, DC, USA. 
Chong, S., Skalka, C., ANd Vaughan, J. A. 2010. Self-identifying sensor data. In Proceedings of the 9th ACM / IEEE International Conference on Information Processing in Sensor Networks. ACM, New York, NY, USA, 82-93.

Chong, S., Skalka, C., AND VAughan, J. A. 2014. Self-identifying data for fair use. Tech. Rep. TR-01-14, Harvard University. Jan. Available at ftp://ftp.deas.harvard.edu/techreports/tr-2014.html.

Cox, I. J. AND MilleR, M. L. 2002. The first 50 years of electronic watermarking. EURASIP Journal on Advances in Signal Processing 2002, 2, 126-132.

CUI, Y. AND WIDOM, J. 2001. Lineage tracing for general data warehouse transformations. In Proceedings of 27th International Conference on Very Large Data Bases. Morgan Kaufmann Publishers, San Francisco, CA.

FRIDRICH, J. AND GOLJAN, M. 1999. Comparing robustness of watermarking techniques. In Security and Watermarking of Multimedia Contents. Vol. 3657. SPIE, Bellingham, WA.

GEHANI, A. AND LINDQVIST, U. 2007. VEIL: A system for certifying video provenance. In Proceedings of the Ninth IEEE International Symposium on Multimedia. IEEE Computer Society, Washington, DC, USA, 263-272.

HARJITO, B., PotdAR, V., AND SINGH, J. 2012. Watermarking technique for copyright protection of wireless sensor network data using LFSR and Kolmogorov complexity. In Proceedings of the 10th International Conference on Advances in Mobile Computing \& Multimedia. ACM, New York, NY, USA, 208-217.

Hubbard Brook Ecosystem Study. http://www.hubbardbrook.org/. Last visited 7/21/14.

JACOBSON, H. I. 1969. The maximum variance of restricted unimodal distributions. The Annals of Mathematical Statistics 40, 5, 1746-1752.

KAmel, I. AND Juma, H. 2011. A lightweight data integrity scheme for sensor networks. Sensors 11, 4, 4118-4136.

Ledlie, J., NG, C., Holland, D. A., Muniswamy-Reddy, K.-K., Braun, U., ANd Seltzer, M. 2005. Provenance-aware sensor data storage. In Proceedings of the 1st IEEE International Workshop on Networking Meets Databases.

LEE, T., BRESSAN, S., AND MADNICK, S. E. 1998. Source attribution for querying against semi-structured documents. In Workshop on Web Information and Data Management. 33-39.

Moreau, L., Clifford, B., Freire, J., Futrelle, J., Gil, Y., Groth, P., Kwasnikowska, N., Miles, S., Missier, P., Myers, J., Plale, B., Simmhan, Y., Stephan, E., AND Den Bussche, J. V. 2011. The Open Provenance Model core specification (v1.1). Future Generation Computer Systems 27, 6, 743756.

PARK, U. AND HeIdemann, J. 2008. Provenance in sensornet republishing. In Proceedings of the 2nd International Provenance and Annotation Workshop. Springer-Verlag, Salt Lake City, Utah, USA, 208-292.

SADRI, F., Ed. 1998. First Workshop on Web Information and Data Management. ACM.

Sagehen Creek Field Station Data Repository. http://sagehen.ucnrs.org/resources.htm. Last visited 8/30/13.

ShenAB, M., Bertino, E., AND GHAFOoR, A. 2008. Watermarking relational databases using optimization-based techniques. IEEE Transactions on Knowledge and Data Engineering 20, 1, 116-129.

Sion, R., Atallah, M., AND Prabhakar, S. 2006. Rights protection for discrete numeric streams. IEEE Transactions on Knowledge and Data Engineering 18, 5, 699-714.

TAN, W. 2003. Containment of relational queries with annotation propagation. In Proceedings of the International Workshop on Database and Programming Languages. Springer-Verlag, Berlin, 37-53.

UbiSec\&Sens Hmac-MD5 Implementation. http://www.ist-ubisecsens.org/downloads/hmac-md5/hmac-md5. php. Last visited 10/29/09.

WIDOM, J. 2004. Trio: A system for integrated management of data, accuracy, and lineage. Technical Report 2004-40, Stanford InfoLab. August. 